Cahiers
de a Recherche
sur les Droits
Fondamentaux

Cahiers de la recherche sur les droits fondamentaux

12 | 2014

Droit et psychiatrie

\title{
Chronique de jurisprudence constitutionnelle française 2013
}

Aurore Catherine, Amandine Cayol, Eugénie Duval, Alexandra Korsakoff et Antoine Siffert

\section{OpenEdition}

12 Journals

Édition électronique

URL : http://journals.openedition.org/crdf/1988

DOI : $10.4000 /$ crdf. 1988

ISSN : 2264-1246

Éditeur

Presses universitaires de Caen

Édition imprimée

Date de publication : 1 décembre 2014

Pagination : 137-152

ISBN : 978-2-84133-507-7

ISSN : 1634-8842

Référence électronique

Aurore Catherine, Amandine Cayol, Eugénie Duval, Alexandra Korsakoff et Antoine Siffert, "Chronique de jurisprudence constitutionnelle française 2013 ", Cahiers de la recherche sur les droits fondamentaux [En ligne], 12 | 2014, mis en ligne le 01 octobre 2015, consulté le 11 février 2020. URL : http:// journals.openedition.org/crdf/1988; DOI : 10.4000/crdf.1988 


\title{
Chronique de jurisprudence constitutionnelle française 2013
}

\author{
Aurore CATHERINE \\ Docteure en droit public, chargée d'enseignement à l'université de Caen Basse-Normandie \\ Centre de recherche sur les droits fondamentaux et les évolutions du droit (CRDFED, EA 2132)
}

\section{Amandine CAYOL}

Maître de conférences en droit privé à l'université de Caen Basse-Normandie

Centre de recherche en droit privé (CRDP, EA 967)

\section{Eugénie DUVAL}

Doctorante en droit public à l'université de Caen Basse-Normandie

Centre de recherche sur les droits fondamentaux et les évolutions du droit (CRDFED, EA 2132)

\section{Alexandra KORSAKOFF}

Doctorante en droit public à l'université de Caen Basse-Normandie

Centre de recherche sur les droits fondamentaux et les évolutions du droit (CRDFED, EA 2132)

\section{Antoine SIFFERT}

Doctorant en droit public à l'université du Havre

Laboratoire de recherche en droits fondamentaux, échanges internationaux et de la mer (Lexfeim)

I. Les modalités du contrôle de constitutionnalité

A. Précisions sur la procédure QPC

1. Une clarification du régime des interventions volontaires

2. De la nature des dispositions contestables

3. De la nature des droits invocables

B. L'articulation de la QPC avec d'autres procédures

1. La première question préjudicielle posée à la Cour de justice de l'Union européenne (CJUE)

2. L'articulation entre le contrôle a priori et le contrôle a posteriori

II. L'évolution du contrôle de constitutionnalité

A. Le droit de la famille

B. Le droit de l'environnement

C. La protection du droit de propriété 


\author{
D. La libre administration des collectivités territoriales \\ 1. L'encadrement de la libre administration des collectivités territoriales par le législateur \\ 2. Les atteintes légitimes à la libre administration des collectivités territoriales \\ 3. Le législateur censuré au nom de la libre administration des collectivités territoriales \\ E. Les garanties de l'article 16 de la Déclaration des droits de I'homme et du citoyen (DDHC)
}

Au cours de l'année 2013, dans le cadre du contrôle de constitutionnalité des lois, le Conseil constitutionnel a rendu vingt-et-une décisions portant sur une loi non encore promulguée et soixante-six décisions à l'occasion d'une question prioritaire de constitutionnalité (QPC). Deux d'entre elles, intervenues dans des domaines isolés, ne font pas l'objet des développements qui suivent bien qu'elles enrichissent la jurisprudence constitutionnelle de manière remarquée. Il s'agit, d'une part, de la décision $\mathrm{n}^{\circ}$ 2013-297 QPC qui donne une définition constitutionnelle du principe de laïcité ${ }^{1}$, et, d'autre part, de la décision $\mathrm{n}^{\circ}$ 2013-318 QPC, première décision sur une QPC par laquelle le Conseil censure le législateur sur le fondement du principe de nécessité des peines ${ }^{2}$. En ce qui concerne les modalités du contrôle de constitutionnalité (I), la quatrième année de contrôle a posteriori donne lieu à des précisions en matière de procédure QPC (A) mais aussi quant à son articulation avec le contrôle $a$ priori des lois et celui de la Cour de justice de l'Union européenne (B). D'un point de vue plus substantiel (II), le Conseil précise sa jurisprudence dans les domaines du droit de la famille (A), du droit de l'environnement (B), de la protection du droit de propriété (C), du principe de la libre administration des collectivités territoriales (D), et enfin des garanties de l'article 16 de la Déclaration des droits de l'homme et du citoyen (DDHC) (E).

\section{Les modalités du contrôle de constitutionnalité}

\section{A. Précisions sur la procédure QPC}

L'année 2013 a avant tout été marquée par la décision $\mathrm{n}^{\circ}$ 2013-322 QPC par laquelle le Conseil constitutionnel est venu préciser l'étendue du contrôle opéré dans le cadre d'une procédure QPC: «il ne lui appartient de procéder à l'interprétation du texte qui lui est déféré que dans la mesure où cette interprétation est nécessaire à l'appréciation de sa constitutionnalité $»^{3}$. Classiquement utilisé dans le cadre du contrôle a priori ${ }^{4}$, il s'agit de la première occurrence de ce considérant de principe dans le cadre du contrôle a posteriori. Outre cet apport majeur, la jurisprudence relative à la procédure applicable aux QPC présente trois principaux intérêts. Elle clarifie le régime des interventions volontaires (1), précise le champ des dispositions contestables (2) ainsi que celui des droits invocables (3).

\section{Une clarification du régime des interventions volontaires}

Ce régime n'a été formalisé que tardivement ${ }^{5}$. Il s'agit de permettre à des tiers de présenter des observations en intervention devant le Conseil dans le cadre des instances QPC. L'article 6 du règlement du 4 février 2010, relatif à la procédure suivie devant le Conseil dans le cadre de la QPC, subordonne cette faculté à une double condition: les tiers doivent justifier d'un intérêt spécial et adresser leurs observations au Conseil constitutionnel dans un délai de trois semaines suivant la date de la transmission de la QPC.

Si la jurisprudence de 2013 n'a pas mis un terme au manque de transparence des critères retenus dans la qualification de cet intérêt spécial ${ }^{6}$, elle apparaît fondamentale sur trois points. D'une part, elle fait montre d'un refus de dévoiement de cet instrument juridictionnel à des fins politiques. Telle est la teneur de la décision $n^{\circ}$ 2013-353 QPC, dans laquelle le Conseil constate que le seul fait qu'un maire soit appelé à mettre en œuvre des dispositions législatives ne saurait caractériser un intérêt spécial, et à ce titre, lui permettre de contester ou défendre

1. CC, déc. no 2013-297 QPC du 21 février 2013, Association pour la promotion et l'expansion de la laïcité, cons. 5: «que le principe de laïcité impose notamment le respect de toutes les croyances, l'égalité de tous les citoyens devant la loi sans distinction de religion et que la République garantisse le libre exercice des cultes; qu'il implique que celle-ci ne salarie aucun culte [...]».

2. CC, déc. $\mathrm{n}^{\circ}$ 2013-318 QPC du 7 juin 2013, M. Mohamed T., cons. 19.

3. CC, déc. $\mathrm{n}^{\circ}$ 2013-322 QPC du 14 juin 2013, M. Philippe W., cons. 12.

4. CC, déc. $n^{\circ}$ 91-298 DC du 24 juillet 1991, Loi portant diverses dispositions d'ordre économique et financier, cons. 33.

5. CC, déc. $\mathrm{n}^{\circ}$ 2011-120 ORGA du 21 juin 2011, Décision modifiant le règlement intérieur sur la procédure suivie devant le Conseil constitutionnel pour les questions prioritaires de constitutionnalité.

6. Ce critère possède des contours flous mais se trouve généralement rempli lorsque la QPC en question porte sur l'abrogation ou le maintien d'une disposition traitant particulièrement de la situation de l'auteur de la demande d'intervention. Si certaines hypothèses ne font pas débat 
ces dispositions dans le cadre d'une intervention QPC?. D'autre part, la recevabilité des mémoires en observation a été subordonnée à une nouvelle condition matérielle: l'existence d'observations sur le bien-fondé de la question posée. Cette exigence découle de la décision $n^{\circ}$ 2013-322 QPC dans laquelle les demandes d'intervention de six associations présentant un intérêt à agir furent rejetées, au motif que ces dernières se réservaient seulement «le droit d'établir [des observations] au vu des mémoires déposés [ultérieurement] par les parties au litige ${ }^{8}$. Enfin, en marge du texte applicable, le Conseil opère une différenciation de régime entre les refus d'admission fondés sur la condition de délai et ceux fondés sur la condition de l'intérêt. À cet égard, il a jugé dans l'affaire no 2013-346 $\mathrm{QPC}^{9} \mathrm{qu}^{\prime} \mathrm{il}$ n'était pas tenu de prendre de décision de non-admission concernant les demandes d'intervention présentées tardivement (c'est-à-dire au-delà du délai de trois semaines requis) ${ }^{10}$.

\section{De la nature des dispositions contestables}

Seule la constitutionnalité de dispositions législatives peut être examinée dans le cadre d'une QPC, c'est-à-dire de lois ordinaires, lois organiques, ordonnances ratifiées ${ }^{11}$ et lois du pays de Nouvelle-Calédonie. Cet examen peut porter sur plusieurs rédactions successives de la disposition contestée $^{12}$, mais aussi sur une pluralité de dispositions législatives à la condition que chacune d'elles dispose d'un lien effectif et concret avec la procédure à laquelle est partie le requérant ${ }^{13}$.
Une difficulté particulière se posa dans l'affaire $\mathrm{n}^{\circ}$ 2013-331 QPC, dans laquelle le Conseil fut saisi d'une QPC portant sur des dispositions législatives partiellement modifiées par une ordonnance non ratifiée. Il convient alors de distinguer suivant que ces dispositions sont séparables ou non. Dans la première hypothèse, les dispositions résultant de l'ordonnance non ratifiée seront purement écartées. Dans la seconde, comme ce fut le cas en l'espèce, leur constitutionnalité ne sera certes pas examinée mais elles seront tout de même prises en compte dans le cadre de l'examen des dispositions législatives ${ }^{14}$.

Si la disposition législative en elle-même peut être contestée, l'interprétation qui en est faite par les juridictions suprêmes peut aussi être contrôlée ${ }^{15}$. En d'autres termes, le Conseil constitutionnel est aussi tenu d'examiner la constitutionnalité de la portée effective qu'une interprétation jurisprudentielle constante confère aux dispositions déférées devant lui. L'année 2013 est ici marquée par un assouplissement tenant à l'évaluation de ce critère de constance, traduisant une extension du contrôle opéré sur les jurisprudences du Conseil d'État et de la Cour de cassation. Traditionnellement, le caractère constant d'une interprétation jurisprudentielle est retenu sur la base de deux principaux critères: d'une part, l'ancienneté de l'interprétation et, d'autre part, sa continuité. Or, si le critère de l'ancienneté est apprécié discrétionnairement par le Conseil, la durée requise tend manifestement à s'écourter, à l'image des treize et dix années respectivement jugées suffisantes dans les décisions n ${ }^{\circ} 2013-336$ QPC $^{16}$ et n ${ }^{\circ} 2013-354$ QPC $^{17}$

(exemples du particulier dont les biens font l'objet d'une procédure d'expropriation à l'occasion d'une QPC portant sur les dispositions fondant cette procédure - CC, déc. $\mathrm{n}^{\circ}$ 2013-338/339 QPC du 13 septembre 2013, Société Invest Hôtels Saint-Dizier Rennes et autre -, ou de collectivités territoriales intervenant dans le cadre de questions portant sur les règles régissant le fonctionnement et la libre administration de ces collectivités - CC, déc. no 2013-303 QPC du 26 avril 2013, Commune de Puyravault; CC, déc. nº 2013-315 QPC du 26 avril 2013 , Commune de Couvrot), d'autres font l'objet de traitements disparates. Il s'agit par exemple des traitements différenciés accordés aux demandes d'intervention provenant d'associations dont les statuts visent la défense des intérêts en cause dans les QPC. Ainsi en 2013, un intérêt spécial fut reconnu à trois associations militant contre le racisme et / ou l'antisémitisme à l'occasion d'une QPC relative au délai de prescription pour les délits de presse à raison de l'origine, l'ethnie, la nation, la race ou la religion (CC, déc. n' 2013-302 QPC du 12 avril 2013, M. Laurent A. et autres), ainsi qu'à deux associations de défense de l'environnement à l'occasion d'une QPC relative à l'interdiction de la fracturation hydraulique pour l'exploration et l'exploitation des hydrocarbures (CC, déc. nº 2013-346 QPC du 11 octobre 2013, Société Schuepbach Energy LL). A contrario, dans cette même décision, les demandes de deux autres associations de défense de l'environnement furent rejetées, sans motivation. Un phénomène similaire est aussi constatable en ce qui concerne les demandes d'intervention émanant d'instances représentatives spécialement intéressées par le maintien ou l'abrogation de dispositions visant les personnes dont elles défendent les intérêts: un intérêt spécial fut par exemple reconnu à l'Association des chambres de commerce et d'industrie des outre-mer dans le cadre d'une QPC relative à la composition du conseil de surveillance des grands ports maritimes des départements d'outre-mer (CC, déc. n 2013-313 QPC du 22 mai 2013, Chambre de commerce et d'industrie de région des îles de Guadeloupe et autres), mais nié à la Confédération française du commerce de gros interentreprises et du commerce international dans le cadre d'une QPC relative aux modalités de recouvrement de la taxe additionnelle à la CVAE (CC, déc. n $2012-298$ QPC du 28 mars 2013 , SARL Majestic Champagne)

7. CC, déc. $\mathrm{n}^{\mathrm{o}} 2013-353$ QPC du 18 octobre 2013, M. Franck $M$. et autres, cons. 1.

8. CC, $\mathrm{n}^{\circ}$ 2013-322 QPC, cons. 3 .

9. $\mathrm{CC}, \mathrm{n}^{\circ} 2013-346 \mathrm{QPC}$.

10. Voir O. Mamoudy, «Précisions sur le régime de l'intervention volontaire et l'invocabilité de la Charte de l'environnement dans le cadre de la QPC», Les petites affiches, ${ }^{\circ}$ 253, 19 décembre 2013, p. 12-22.

11. Si les dispositions d'une ordonnance ratifiée peuvent être examinées (CC, déc. n 2012-28o QPC du 12 octobre 2012, Société Groupe Canal Plus et autre), tel n'est pas le cas pour celles des ordonnances non ratifiées (CC, déc. nº 2011-219 QPC du 10 février 2012, M. Patrick É., cons. 3).

12. CC, déc. $\mathrm{n}^{\circ}$ 2013-300 QPC du 5 avril 2013, Chambre de commerce et d'industrie de Brest.

13. CC, déc. $n^{\circ} 2013-334 / 335$ QPC du 26 juillet 2013, Société Somaf et autre, cons. 5.

14. CC, déc. $n^{\circ}$ 2013-331 QPC du 5 juillet 2013, Société Numéricâble SAS et autre, cons. 3.

15. CC, déc. $\mathrm{n}^{\circ}$ 2010-39 QPC du 6 octobre 2010, $M^{\text {mes }}$ Isabelle D. et Isabelle B.; CC, déc. $\mathrm{n}^{\circ}$ 2010-52 QPC du 14 octobre 2010 , Compagnie agricole de la Crau. Seule l'interprétation constante des juridictions suprêmes peut être analysée: CC, déc. no 2011-120 QPC du 8 avril 2011, M. Ismaël A., cons. 9.

16. CC, déc. $\mathrm{n}^{\circ}$ 2013-336 QPC du $1^{\mathrm{er}}$ août 2013, Société Natixis Asset Management.

17. CC, déc. nº 2013-354 QPC du 22 novembre 2013, $M^{\text {me }}$ Charly K. 
(une interprétation s'éloignant fortement des premières décisions du Conseil en la matière $\left.{ }^{18}\right)$. Quant au critère de la continuité de l'interprétation, il fut déjà fortement relativisé en 2011 lorsque le juge de Montpensier jugea que la seule interprétation donnée dans la décision de renvoi était susceptible de constituer une jurisprudence constante, et ce même en l'absence de décision antérieure confirmant cette interprétation ${ }^{19}$. L'assouplissement de ce critère a été poursuivi en 2013 lorsque le Conseil accepta de contrôler une jurisprudence non homogène, c'est-àdire connaissant des exceptions. Dans l'affaire no $2013-311$ $\mathrm{QPC}^{20}$, le juge constitutionnel a en effet reconnu valeur de jurisprudence constante à l'interprétation retenue par la formation plénière de la Cour de cassation de l'article 53 de la loi du 29 juillet 1881 sur la liberté de la presse, eu égard à son autorité et nonobstant la divergence d'interprétation avec la première chambre civile.

\section{De la nature des droits invocables}

La nature des droits invocables dans le cadre d'une QPC a aussi été précisée cette année, sans grand bouleversement toutefois. Tout d'abord, deux dispositions ont été jugées non invocables car n'instituant pas un droit ou une liberté. Il s'agit, d'une part, du $5^{\circ}$ du paragraphe II de l'article 34 de la loi organique relative aux lois de finances $\mathrm{du} 1^{\mathrm{er}}$ août 2001 (qui dispose que «dans la seconde partie, la loi de finances de l'année autorise l'octroi des garanties de l'État et fixe leur régime " ${ }^{21}$ et, d'autre part, de l'alinéa 2 de l'article 72 de la Constitution (suivant lequel «les collectivités territoriales ont vocation à prendre les décisions pour l'ensemble des compétences qui peuvent le mieux être mises en œuvre à leur échelon $»)^{22}$. S'il est vrai que le principe de libre administration des collectivités territoriales est régulière- ment invoqué dans le cadre d'une QPC depuis $2010^{23}$, le Conseil constitutionnel en a donc supprimé l'opportunité concernant le principe de subsidiarité dont il avait d'ailleurs déjà restreint la portée dans le cadre du contrôle $a$ priori ${ }^{24}$.

Une précision essentielle a été apportée concernant le moyen tiré de l'incompétence négative du législateur. Depuis sa célèbre jurisprudence SNC Kimberley Clark de $2010^{25}$, ce moyen n'est opérant que lorsque l'incompétence négative "affecte» un droit ou une liberté au sens de l'article 61-1 de la Constitution ${ }^{26}$. Dans ce cadre, le Conseil constitutionnel n'accepte d'examiner les griefs selon lesquels une disposition législative ne traite pas d'une situation que lorsque cette dernière ne fait l'objet d'aucune autre disposition législative. À défaut, le moyen tiré de l'article 34 est inopérant ${ }^{27}$.

D’autre part, plusieurs précisions ont été apportées concernant les principes fondamentaux reconnus par les lois de la République (PFRLR). Il doit être souligné ici que le Conseil constitutionnel n'accepte de recourir à la notion qu'à titre subsidiaire, lorsque leur contenu n'est pas susceptible de trouver un fondement direct dans les textes constitutionnels. Si ce principe avait déjà été affirmé en $2006^{28}$, le Conseil a cette année opéré une substitution du fondement constitutionnel de la liberté de conscience. Si cette dernière est une liberté invocable dans le cadre de la QPC, cela résulte de son attachement à l'article 10 de la Déclaration de 1789 et au cinquième alinéa du préambule de $1946^{29}$, et non de sa consécration au titre des PFRLR depuis $1977^{30}$. On peut aussi noter en 2013 le premier examen de la conformité d'une disposition législative déjà promulguée au regard du PFRLR de la justice pénale des mineurs ${ }^{31}$, ainsi que le refus d'ériger l'altérité sexuelle des époux ${ }^{32}$ et l'autonomie des chambres de commerce et de l'industrie ${ }^{33}$ au rang de PFRLR.

18. À titre d'exemples: CC, déc. n² 2010-96 QPC du 4 février 2011, M. Jean-Louis de L. (contrôle d'une jurisprudence de la Cour de cassation datant de 1965); CC, déc. $\mathrm{n}^{\circ}$ 2011-113/115 QPC du $1^{\mathrm{er}}$ avril 2011, M. Xavier P. et autre (contrôle d'une jurisprudence de la Cour de cassation datant de 1963); CC, déc. nº 2011-127 QPC du 6 mai 2011, Consorts C. (contrôle d'une jurisprudence de la Cour de cassation datant de 1979).

19. CC, déc. $\mathrm{n}^{\circ}$ 2011-185 QPC du 21 octobre 2011, M. Jean-Louis C.; jurisprudence reprise dans CC, déc. no 2013-340 du 20 septembre 2013, M. Alain G.

20. CC, déc. n 2013-311 QPC du 17 mai 2013, Société ÉCOCERT France.

21. CC, déc. n 2013-344 QPC du 27 septembre 2013, Société SCOR SE, cons. 9.

22. CC, déc. $\mathrm{n}^{\circ}$ 2013-304 QPC du 26 avril 2013, Commune de Maing, cons. 7.

23. Principe invocable dans le cadre d'une QPC depuis: CC, déc. nº 2010-12 QPC du 2 juillet 2010, Commune de Dunkerque, cons. 4; régulièrement invoqué depuis. Quelques exemples de l'année 2013: CC, déc. nº 2013-315 QPC du 26 avril 2013; CC, déc. n 2013-305/306/307 QPC du 19 avril 2013, Commune de Tourville-la-Rivière.

24. CC, déc. n² 2005-516 DC du 7 juillet 2005, Loi de programme fixant les orientations de la politique énergétique, cons. 12.

25. CC, déc. $\mathrm{n}^{\circ}$ 2010-5 QPC du 18 juin 2010, SNC Kimberly Clark.

26. Cette limite a été récemment relativisée par une partie de la doctrine qui tend à considérer, au regard «des statistiques flatteuses» de l'année 2013, que l'incompétence négative tendrait à "se suffir à elle-même» car mettant toujours plus ou moins directement en jeu un droit ou une liberté garanti par la Constitution. En ce sens, lire: D. Rousseau, P.-Y. Gahdoun, J. Bonnet, "Chronique de jurisprudence constitutionnelle 2013 ", Revue du droit public, $\mathrm{n}^{\circ} 1,2014$, p. 207-245. Quatre censures sur le fondement de l'article 34 ont en effet été opérées en 2013: déc. n²013-336 QPC; déc. $\mathrm{n}^{\circ}$ 2012-298 QPC (à noter: première application à une loi fiscale dans le cadre du contrôle a posteriori); déc. nº 2013-343 QPC du 27 septembre 2013, Époux L.; déc. n 2013-351 QPC du 25 octobre 2013, Société Boulanger.

27. CC, déc. $\mathrm{n}^{\circ}$ 2013-312 QPC du 22 mai 2013, M. Jory Orlando T.; CC, déc. nº 2013-358 QPC du 29 novembre 2013, M. Azdine A.

28. À propos des droits de la défense, érigés au titre de PFRLR par CC, déc. $\mathrm{n}^{\circ}$ 76-70 DC du 2 décembre 1976, Loi relative au développement de la prévention des accidents du travail, cons. 2; puis rattachés à l'article 16 de la DDHC dans CC, déc. no 2006-535 DC du 30 mars 2006, Loi pour l'égalité des chances, cons. 24.

29. CC, déc. $\mathrm{n}^{\circ}$ 2013-353 QPC.

30. CC, déc. $\mathrm{n}^{\circ} 77-87 \mathrm{DC}$ du 23 novembre 1977, Loi relative à la liberté d'enseignement, cons. 5 .

31. CC, déc. $\mathrm{n}^{\circ}$ 2013-356 QPC du 29 novembre 2013, M. Christophe D., cons. 14.

32. CC, déc. $\mathrm{n}^{\circ}$ 2013-669 DC du 17 mai 2013, Loi ouvrant le mariage aux couples de personnes de même sexe, cons. 21.

33. CC, déc. $\mathrm{n}^{\circ}$ 2013-313 QPC, cons. 6. 
Enfin, malgré plusieurs requêtes invoquant la méconnaissance de la Charte de l'environnement, la jurisprudence de l'année ne fait preuve d'aucune innovation en la matière. En effet, si le Conseil a confirmé l'invocabilité du principe de participation du public tiré de son article $7^{34}$, contrairement à son article $6^{35}$, le suspense demeure en ce qui concerne le principe de précaution, posé à l'article 5 , le moyen ayant été jugé inopérant dans le cas d'espèce où il fut soulevé ${ }^{36}$.

\section{B. L'articulation de la QPC avec d'autres procédures}

La jurisprudence du Conseil constitutionnel est particulièrement innovante sur la question: en premier lieu en raison de la première question préjudicielle que le Conseil pose à la Cour de justice de l'Union européenne (CJUE) (1); en second lieu parce qu'il vient préciser certains aspects de l'articulation entre son contrôle a priori et son contrôle a posteriori du contrôle de constitutionnalité de la loi (2).

\section{La première question préjudicielle posée à la Cour de justice de I'Union européenne (CJUE)}

C'est une des innovations majeures de la jurisprudence constitutionnelle de l'année 2013. Pour la première fois dans son histoire, le Conseil constitutionnel a saisi la CJUE d'une question préjudicielle ${ }^{37}$, opérant ainsi un revirement dans sa jurisprudence. En effet, a priori, le système français de contrôle de constitutionnalité de la loi ne permettait pas de recourir à la $\mathrm{CJUE}^{38}$. C'est en revenant sur ce principe que le Conseil constitutionnel fait, dans sa décision $n^{\circ} 2013-314$ P QPC, œuvre innovante. En l'espèce, le requérant a formé devant la Cour de cassation un pourvoi assorti d'une QPC estimant que le quatrième alinéa de l'article 695-46 du Code de procédure pénale selon lequel la chambre de l'instruction statue «sans recours » portait atteinte au principe d'égalité devant la justice et au droit à un recours juridictionnel effectif. Dans sa décision $\mathrm{n}^{\circ}$ 2013-314 P QPC, le Conseil constitutionnel, considérant que la question posait des interrogations sérieuses sur la portée du délai prévu aux articles 27 et 28 de la décision-cadre $n^{\circ}$ 2002/584/JAI du Conseil du 13 juin 2002 relative au mandat d'arrêt européen et aux procédures de remise entre États membres, a estimé qu'une décision sur l'interprétation de cette décision-cadre était nécessaire pour lui permettre d'exercer son contrôle. En d'autres termes, la décision-cadre relative au mandat d'arrêt européen ayant posé qu'il devait être statué «au plus tard trente jours après réception " d'une demande d'extension avait-elle été transposée correctement par la loi française qui dispose que «la chambre d'instruction statue sans recours dans un délai d'un mois à compter de la réception de la demande»? C'est en ce sens que le Conseil constitutionnel a saisi la CJUE et sursis à statuer dans l'attente de sa décision lui demandant de se prononcer dans le cadre de la procédure d'urgence, en invoquant à cette fin un certain nombre d'arguments (cons. 8). En premier lieu, il a fait valoir qu'il est possible qu'une telle saisine de la Cour conduise au dépassement du délai de trois mois dans lequel il doit statuer en application de l'article 23-10 de l'ordonnance $\mathrm{n}^{\circ}$ 58-1067 du 7 novembre 1958 portant loi organique sur le Conseil constitutionnel. En deuxième lieu, il précise que le requérant est détenu - ce qui fait entrer la question préjudicielle dans le dernier alinéa de l'article 267 du TFUE qui dispose que, si la question est soulevée dans une affaire pendante devant une juridiction nationale concernant une personne détenue, la Cour se prononce «dans les plus brefs délais». Enfin, pour l'examen des renvois préjudiciels relatifs à l'espace de liberté, de sécurité et de justice, l'article 23 bis du statut de la CJUE prévoit la possibilité d'une procédure d'urgence. Par cette demande et en obtenant gain de cause auprès de la CJUE, le Conseil constitutionnel fait « œuvre créatrice» puisqu'il «redéfinit [ainsi] le cadre procédural» posé par le législateur organique: «il prononce un sursis à statuer, ce qui n'est prévu par aucun texte [et] il se libère du respect du délai de trois mois pour juger une QPC ${ }^{39}$. La CJUE a fait droit à la demande de procédure d'urgence en statuant sur la question préjudicielle le 30 mai 2013. La Cour a jugé que l'interdiction de tout recours résultant de l'article 695-46 du Code de procédure pénale ne trouvait aucune justification dans le droit de l'Union européenne. Le Conseil constitutionnel a en conséquence déclaré contraires à la Constitution les mots «sans recours» de la disposition litigieuse. Ces «lectures convergentes ${ }^{40}$ des deux juges, qui

34. CC, déc. n 2013-317 QPC du 24 mai 2013, Syndicat français de l'industrie cimentière et autre, cons. 5 (jurisprudence constante depuis CC, déc. no 2011-183/184 QPC du 14 décembre 2011, Association France Nature Environnement, cons. 6).

35. CC, déc. $\mathrm{n}^{\circ}$ 2013-346 QPC, cons. 19 (confirmation de CC, déc. $\mathrm{n}^{\circ}$ 2012-283 QPC du 23 novembre 2012, M. Antoine de M., cons. 22).

36. CC, déc. nº 2013-346 QPC, cons. 20.

37. La CJUE est en effet compétente, en vertu de l'article 267 du Traité sur le fonctionnement de l'Union européenne (TFUE), «pour statuer, à titre préjudiciel: b) sur la validité et l'interprétation des actes pris par les institutions, organes ou organismes de l'Union".

38. Dans sa décision $\mathrm{n}^{\circ}$ 2006-543 DC du 30 novembre 2006, Loi relative au secteur de l'énergie, le Conseil affirmait en ce sens que, «devant statuer avant la promulgation de la loi dans le délai prévu par l'article 61 de la Constitution, il ne peut saisir la Cour de justice des Communautés européennes de la question préjudicielle prévue par l'article 234 du Traité instituant la Communauté européenne et qu'en tout état de cause, il revient aux autorités juridictionnelles nationales, le cas échéant, de saisir la Cour de justice des Communautés européennes à titre préjudiciel».

39. D. Rousseau, P.-Y. Gahdoun, J. Bonnet, «Chronique de jurisprudence constitutionnelle 2013», p. 242.

40. H. Labayle, R. Medhi, «Le droit au juge et le mandat d'arrêt européen : lectures convergentes de la Cour de justice de l'Union européenne et du Conseil constitutionnel ", Revue française de droit administratif, $n^{\circ} 4,2013$, p. 691-708. 
permettent de parler de «dialogue des juges ${ }^{41}$, conduisent certains auteurs à évoquer une « européanisation du juge constitutionnel ${ }^{42}$. Dominique Rousseau évoque le fait que «le contentieux européen devient, avec la décision du 4 avril 2013, un moment du contentieux constitutionnel ${ }^{43}$. Un autre aspect de cette décision mérite d'être relevé: en faisant usage de l'article 267 du Traité de Lisbonne selon lequel les questions préjudicielles sont transmises à la CJUE par les juridictions nationales des États, c'est à une consolidation de sa nature juridictionnelle que le Conseil constitutionnel procède.

\section{L'articulation entre le contrôle a priori et le contrôle a posteriori}

Certaines décisions que le Conseil constitutionnel a rendues en 2013 mettent en avant les différences existantes entre le contrôle a priori et le contrôle a posteriori de constitutionnalité de la loi.

Dans sa décision $n^{\circ}$ 2013-334/335 QPC relative à la loi no 2004-639 du 2 juillet 2004 sur l'octroi de mer, le Conseil vient rappeler une première divergence avec le contrôle a priori : il ne peut se prononcer, dans le cadre de la procédure QPC, sur l'intégralité d'une loi. Précisément, dans le cinquième considérant, le Conseil confirme la possibilité d'invoquer dans une seule QPC la non-conformité de plusieurs dispositions législatives, mais il précise la nécessité que chaque disposition contestée soit en lien direct avec le litige. Il est également impératif d'indiquer avec précision les droits et libertés qui ne seraient pas respectés par le texte. Le Conseil constitutionnel rappelle le rôle de filtre dévolu au Conseil d'État et à la Cour de cassation dans cette procédure. Ils sont tenus

[...] de vérifier que chacune des dispositions législatives visées par la question est applicable au litige puis, au regard de chaque disposition législative retenue comme applicable au litige, que la question est nouvelle ou présente un caractère sérieux.

N'étant pas, dans cette affaire, saisi conformément à ces diverses exigences, le Conseil considère qu'il n'y a pas lieu de statuer sur les deux QPC jointes en l'espèce.

Par ailleurs, le juge constitutionnel est venu, cette année encore, préciser la jurisprudence issue de sa décision $\mathrm{n}^{\circ}$ 85-187 DC, État d'urgence en Nouvelle-Calédonie ${ }^{44}$. Selon cette jurisprudence, «la conformité à la Consti- tution d'une loi déjà promulguée peut être appréciée à l'occasion de l'examen des dispositions législatives qui la modifient, la complètent ou affectent son domaine ${ }^{45}$. La question de l'articulation du contrôle a priori avec le contrôle a posteriori se pose toujours avec autant d'acuité. Précisément, l'importance du contrôle $a$ priori « semblait devoir décliner du fait même de l'institution du contrôle a posteriori ${ }^{46}$. Le Conseil constitutionnel a, dans ce contexte, été amené à renouveler en 2013 sa jurisprudence dite «néo-calédonienne» en ayant recours à une conception relativement large de la condition relative à la modification, au complément ou à l'affectation du domaine de la loi promulguée par la loi soumise au contrôle de constitutionnalité, prolongeant ainsi les décisions qu'il a rendues sur la question en 2012. L'objectif poursuivi est toujours le même: purger l'ordre juridique des dispositions législatives entrées en vigueur mais inconstitutionnelles. Dans sa décision $n^{\circ}$ 2013-667 DC, le Conseil a considéré que relevait de cette catégorie de lois en vigueur pouvant être contrôlées à l'occasion d'une loi déférée le tableau $n^{\circ} 2$ existant annexé au Code électoral fixant la répartition des conseillers de Paris, dès lors que l'article 30 de la loi relative à l'élection des conseillers départementaux, des conseillers municipaux et des conseillers communautaires et modifiant le calendrier électoral abrogeait et remplaçait ce tableau par un nouveau tableau annexé à cette loi. Il en a jugé de même dans sa décision n 2013-669 DC des articles L. 225-2 et 225-17 du Code de l'action sociale et des familles relatifs à l'agrément des personnes qui désirent accueillir un enfant en vue de l'adoption, dès lors que la loi ouvrant le mariage aux personnes de même sexe, en permettant à ces couples d'adopter un enfant, affectait le domaine d'application de ces dispositions. Dans sa décision n ${ }^{\circ}$ 2013-672 DC, le Conseil considère que les dispositions contestées du $2^{\circ}$ du paragraphe II de l'article 1 de la loi relative à la sécurisation de l'emploi déférée complètent celles de l'article L. 912-1 du Code de la sécurité sociale. Il juge que si, par les dispositions de l'article L. 912-1 du Code de la sécurité sociale, le législateur a entendu faciliter l'accès de toutes les entreprises d'une même branche à une protection complémentaire et assurer un régime de mutualisation des risques, poursuivant ainsi un but d'intérêt général, elles méconnaissent toutefois la liberté contractuelle et la liberté d'entreprendre. L'article L. 912-1 du Code de la sécurité sociale, tel qu'il a été introduit dans l'ordonnancement juridique par la loi du

41. M. Disant, "Question (préjudicielle) sur question (prioritaire) - Célébration ou mirage du “dialogue des juges" ?», Gazette du palais, 18 octobre 2013, p. 18-19; J. Roux, «Premier renvoi préjudiciel du Conseil constitutionnel à la Cour de Justice et conjonction de dialogues des juges autour du mandat d'arrêt européen", Revue trimestrielle de droit européen, n 3, 2013, p. 531-557.

42. F. Chaltiel Terral, «L'européanisation du juge constitutionnel: le dialogue renforcé entre le Conseil constitutionnel et la Cour de Justice de l'Union européenne", Les petites affiches, $\mathrm{n}^{\circ}$ 129, 28 juin 2013, p. 4-17.

43. D. Rousseau, «L'intégration du conseil constitutionnel au système juridictionnel européen », Gazette du palais, 5 mai 2013 , p. 1603.

44. CC, déc. $\mathrm{n}^{\circ}$ 85-187 DC du 25 janvier 1985, Loi relative à l'état d'urgence en Nouvelle-Calédonie et dépendances.

45. CC, déc. n 2012-654 DC du 9 août 2012, Loi de finances rectificative pour 2012, cons. 83; CC, déc. nº $2012-656$ DC du 24 octobre 2012 , Loi portant création des emplois d'avenir, cons. 17; CC, déc. n² 2012-659 DC du 13 décembre 2012, Loi de financement de la sécurité sociale pour 2013, cons. 20; CC, déc. n 2012-662 DC du 29 décembre 2012, Loi de finances pour 2013, cons. 20.

46. B. Genevois, «Un exemple de l'influence du contrôle a posteriori sur le contrôle a priori: l'application de la jurisprudence État d'urgence en Nouvelle-Calédonie», Revue française de droit administratif, nº 1, 2013, p. 2. 
8 août 1994 relative à la protection sociale complémentaire, est ainsi censuré. En effet, le fait que l'entreprise soit liée avec un cocontractant déjà désigné par un contrat négocié au niveau de la branche et au contenu totalement prédéfini et le fait que les entreprises de cette branche se trouvent liées avec l'organisme de prévoyance désigné par l'accord, alors même qu'antérieurement à celui-ci elles étaient liées par un contrat conclu avec un autre organisme, portent une atteinte disproportionnée aux droits en cause. Le $2^{\circ}$ du paragraphe II de l'article 1 de la loi déférée complétant ces dispositions est ainsi de la même façon déclaré inconstitutionnel. Il est intéressant de noter que l'article L. 912-1 du Code de la sécurité sociale avait pourtant été jugé conforme au droit de l'Union européenne ${ }^{47}$ par le Conseil d'État ${ }^{48}$ et la CJUE ${ }^{49}$.

Cette décision est par ailleurs importante dans la mesure où le Conseil y règle les effets dans le temps d'une telle déclaration d'inconstitutionnalité sécurisant ainsi l'ordre juridique. Alors que dans la décision n 2013-667 DC, la censure prend effet immédiatement, dans la décision n ${ }^{\circ}$ 2013-672 DC «la déclaration d'inconstitutionnalité de l'article L. 912-1 du Code de la sécurité sociale prend effet à compter de la publication de la présente décision » mais

[...] n'est toutefois pas applicable aux contrats pris sur ce fondement, en cours lors de cette publication, et liant les entreprises à celles qui sont régies par le code des assurances, aux institutions relevant du titre III du code de la sécurité sociale et aux mutuelles relevant du code de la mutualité.

Aucune nouvelle convention ne doit désormais être passée sur le fondement de ces dispositions. Les conventions déjà conclues continueront en revanche de produire leurs effets jusqu'à leur terme prévu initialement, ce qui va dans le sens d'une stabilité des relations contractuelles. Cette décision permet à Anne-Laure Cassard-Valembois d'écrire que:

Le contrôle des lois promulguées, qu'il s'opère sur le fondement de l'article 61 ou de l'article 61-1 de la Constitution, s'exerce ainsi dans des conditions et des formes qui se ressemblent de plus en plus ${ }^{50}$.

La disposition législative entrée en vigueur mais déclarée inconstitutionnelle est, dans l'attente de son abrogation par le Parlement, neutralisée. Elle n'a plus de force normative. Dans la décision n 2013-349 QPC, saisi à nouveau pour statuer sur la constitutionnalité de l'article L. 912-1 du Code de la sécurité sociale, le Conseil précise que la

[...] déclaration d'inconstitutionnalité prend effet à compter de la publication de sa décision; qu'il suit de là qu'à compter de cette date de publication, soit le 16 juin 2013, l'article L. 912-1 du code de la sécurité sociale ne peut plus être appliqué 51 .

Puis il ajoute, dans le considérant suivant, que, lorsque la déclaration d'inconstitutionnalité «néo-calédonienne» est reportée dans le temps, les dispositions déclarées inconstitutionnelles ne peuvent pas faire l'objet d'une QPC pour la période pendant laquelle elles demeurent applicables. En revanche, la disposition législative contestée est, pour des litiges relatifs à la période antérieure à cette date, susceptible d'être invoquée ou appliquée devant les juridictions. Cette décision est particulièrement importante en ce qu'elle traduit une lecture plus large du Conseil de l'article 62 de la Constitution que celle qu'il fait traditionnellement. Il étend en effet incontestablement l'autorité des décisions qu'il rend, ce que l'on retrouve d'ailleurs dans la décision no 2013-682 DC dans laquelle l'autorité de l'article 62 s'applique également aux dispositions d'une loi qui, «bien que rédigées sous une forme différente, ont, en substance, un objet analogue à celui des dispositions législatives déclarées contraires à la Constitution».

\section{L'évolution du contrôle de constitutionnalité}

\section{A. Le droit de la famille}

Plusieurs décisions rendues en 2013 concernent le mariage. Si l'on pense immédiatement à la très médiatique décision $n^{\circ}$ 2013-669 DC relative à la loi ouvrant le mariage aux couples de même sexe, tel est également le cas des décisions $n^{\circ}$ 2013-324 QPC ${ }^{52}$ et $n^{0}$ 2013-330 QPC ${ }^{53}$. Ces dernières mettent de nouveau en exergue les spécificités des conséquences découlant du mariage. Depuis 2010, le Conseil insiste sur la différence existant entre les couples selon qu'il s'agit de concubins, de partenaires d'un pacte civil de solidarité (pacs), ou de personnes mariées ${ }^{54}$. Seul le mariage comporte un engagement dans la durée. Même

47. Voir A.-L. Cassard-Valembois, «L'utilisation renouvelée de la jurisprudence "État d'urgence en Nouvelle-Calédonie" au profit de la liberté contractuelle et de la liberté d'entreprendre", Constitutions, $\mathrm{n}^{\circ}$ 3, 2013, p. 405: "La décision n 2013-672 DC illustre ainsi le fait que, bien que bénéficiant d'une faible protection constitutionnelle, la liberté d'entreprendre et la liberté contractuelle ne sont pas pour autant inefficaces: elles permettent en l'espèce de censurer un dispositif législatif qui avait été jugé conforme aux règles européennes de la concurrence, dont la rigueur n'est pourtant plus à démontrer».

48. CE, 19 mai $2008, n^{\circ} 298907$.

49. CJUE, 3 mars 2011, aff. C-437/o9.

50. A.-L. Cassard-Valembois, «L'utilisation renouvelée...», p. 400.

51. CC, déc. $\mathrm{n}^{\circ}$ 2013-349 QPC du 18 octobre 2013, Sociétés Allianz IARD et autres, cons. 2.

52. CC, déc. $\mathrm{n}^{\circ}$ 2013-324 QPC du 21 juin 2013, $M^{\text {me }}$ Micheline $L$.

53. CC, déc. $n^{\circ}$ 2013-330 QPC du 28 juin 2013, $M^{\text {me }}$ Nicole $B$.

54. Voir A. Cayol, «Question prioritaire de constitutionnalité et droit de la famille», Cahiers de la recherche sur les droits fondamentaux, ${ }^{\circ}$ 11, 2013, p. 17. 
après le décès d'un des époux, certains effets du mariage subsistent, notamment du fait de la vocation successorale du conjoint survivant. Propre au mariage, cette règle n'est pas étendue aux autres formes de couples. Encore faut-il toutefois que les liens du mariage n'aient pas été rompus. Les situations du conjoint survivant et du conjoint divorcé sont bien distinctes. C'est ce qu'a (sans surprise!) affirmé le Conseil constitutionnel dans ces deux décisions. Le principe d'égalité n'impose donc pas que le conjoint divorcé perçoive la pension militaire d'invalidité au même titre que le conjoint survivant ${ }^{55}$. Parallèlement, les avantages susceptibles de résulter d'une séparation volontaire ne peuvent être étendus aux personnes veuves. Tel est notamment le cas de la décharge de solidarité en matière fiscale. Prévue dans l'hypothèse d'une rupture de la vie commune entraînant une disproportion flagrante entre la dette fiscale du couple (dont les deux membres sont en principe solidairement tenus) et la situation financière et patrimoniale d'un des conjoints, cette décharge joue à la suite d'un divorce ou de la rupture d'un pacs. Elle ne saurait jouer à l'occasion du décès d'un des époux, le conjoint survivant étant alors héritier du défunt. Dès lors, «en raison de sa situation financière et patrimoniale ainsi que des modalités selon lesquelles les créances fiscales du couple peuvent être recouvrées ${ }^{56}$, le conjoint survivant ne se trouve pas, au regard de l'objet de la loi, dans une situation identique à celle d'une personne divorcée ou séparée ${ }^{57}$.

La décision la plus marquante de l'année concernant le mariage reste indéniablement la décision $\mathrm{n}^{\circ}$ 2013-669 DC, dans laquelle le Conseil déclare conforme à la Constitution la loi ouvrant le mariage aux couples de même sexe ${ }^{58}$. Il rejette pour ce faire l'existence d'un principe fondamental reconnu par les lois de la République, selon lequel le mariage supposerait une condition d'altérité sexuelle des époux. Les PFRLR sont réaffirmés par le préambule de la Constitution de 1946, lequel n'en dresse toutefois aucune liste. Le Conseil affirme, depuis sa décision du 16 juillet 1971 relative à la liberté d'association ${ }^{59}$ qu'ils font partie du bloc de constitutionnalité. Moins d'une dizaine de PFRLR ont été reconnus depuis ${ }^{60}$. Trois conditions sont traditionnellement requises : il doit s'agir de principes fondamentaux, inscrits dans une loi de la République antérieure à 1946, et qui n'ont jamais été remis en cause. La Cour de cassation a affirmé que le Code civil prévoit qu'un mariage ne peut concerner qu'un couple composé d'un homme et d'une femme ${ }^{61}$. Le Code civil est antérieur à la Constitution de 1946 et la condition d'altérité sexuelle n'a fait l'objet d'aucune exception par la suite. L'argumentation selon laquelle l'ouverture du mariage aux couples homosexuels serait contraire à un PFRLR semblait donc sérieuse $^{62}$. Toutefois, dans sa décision no 2013-669 DC, le Conseil affirme qu'il n'en est rien:

[...] cette règle qui n'intéresse ni les droits et libertés fondamentaux, ni la souveraineté nationale, ni l'organisation des pouvoirs publics, ne peut constituer un principe fondamental reconnu par les lois de la République au sens du premier alinéa du Préambule de $1946^{63}$.

Le Conseil pose, pour la première fois, une condition supplémentaire à l'existence d'un PFRLR, relative au domaine matériel du principe concerné. Ceci a pu être critiqué, comme révélateur de la volonté de valider à tout prix la loi déférée ${ }^{64}$. Toutefois, même si elle n'avait jamais été clairement énoncée jusque-là, cette condition n'est pas véritablement nouvelle. Tous les PFRLR précédemment consacrés concernaient en effet soit l'organisation des pouvoirs publics ${ }^{65}$, soit les droits et libertés fondamentaux ${ }^{66}$. Si la liberté du mariage relève certes des droits et

55. CC, déc. $\mathrm{n}^{\circ}$ 2013-324 QPC, cons. 5: « d'autre part, le conjoint survivant et le conjoint divorcé se trouvent dans des situations différentes; [...] ni le principe d'égalité, ni aucune autre exigence constitutionnelle n'imposent d'octroyer au conjoint divorcé le bénéfice d'une pension accordée au conjoint survivant».

56. En cas de divorce ou de séparation, la décharge de solidarité conduit seulement en pratique à diviser la dette entre les deux anciens membres du couple. Au contraire, en cas de décès, la décharge créerait un risque de non-recouvrement d'une partie de la dette, en l'absence d'autre héritier ayant accepté la succession.

57. CC, déc. $\mathrm{n}^{\circ}$ 2013-330 QPC, cons. 4 .

58. Il faut noter que la décision $\mathrm{n}^{\circ}$ 2013-353 QPC a refusé de reconnaître une clause de conscience aux maires (cons. 10). La Cour européenne des droits de l'homme vient d'être saisie le 24 février 2014 par treize maires français à la suite de ce refus.

59. CC, déc. $\mathrm{n}^{\circ} 71-44$ DC du 16 juillet 1971, Loi complétant les dispositions des articles 5 et 7 de la loi du $1^{\text {er }}$ juillet 1901 relative au contrat d'association (Liberté d'association).

60. Liberté d'enseignement (CC, déc. n 77-87 DC du 23 novembre 1977, Loi complémentaire à la loi no 59 -1557 du 31 décembre 1959 modifiée par la loi $n^{\circ} 71-400 \mathrm{du} 1^{\text {er }}$ juin 1971 et relative à la liberté de l'enseignement), liberté de conscience (dans la même décision), indépendance de la juridiction administrative (CC, déc. no 80-119 DC du 22 juillet 1980, Loi portant validation d'actes administratifs), compétence exclusive de la juridiction administrative en matière d'annulation d'actes administratifs (CC, déc. no 86-224 DC du 23 janvier 1987, Loi transférant à la juridiction judiciaire le contentieux des décisions du Conseil de la concurrence), libertés universitaires (CC, déc. nº 83-165 DC du 20 janvier 1984, Loi relative à l'enseignement supérieur), justice pénale des mineurs (CC, déc. nº 2002-461 DC du 29 août 2002, Loi d'orientation et de programmation pour la justice).

61. Cass., $1^{\text {re }}$ civ., 19 décembre 2007 , pourvoi no $06-21369$.

62. L'existence d'un PFRLR concernant «le principe de valeur constitutionnelle de la filiation binaire» l'était beaucoup moins. Comme le souligne le Conseil, la législation antérieure à 1946 «a toujours compris des règles limitant ou encadrant les conditions dans lesquelles un enfant peut voir établir les liens de filiation à l'égard du père ou de la mère dont il est issu » (CC, déc. nº 2013-669 DC, cons. 56).

63. CC, déc. $\mathrm{n}^{\circ}$ 2013-669 DC, cons. 21.

64. Voir A.-M. Le Pourhiet, «Le mariage de Mamère et la "Constitution de mon père" ", Constitutions, n 2, 2013, p. 166-170; A. Roblot-Troizier, «La loi et les principes fondamentaux reconnus par les lois de la République», Revue française de droit administratif, n 5, 2013 , p. 945-951.

65. Indépendance de la juridiction administrative, compétence exclusive de la juridiction administrative en matière d'annulation d'actes administratifs, justice pénale des mineurs.

66. Liberté d'enseignement, liberté de conscience, libertés universitaires. 
libertés fondamentaux ${ }^{67}$, l'altérité sexuelle des époux ne la concerne pas directement. Comme l'avait précisé le Conseil dans sa décision $\mathrm{n}^{\circ}$ 2010-92 QPC, la liberté du mariage n'empêche ni le législateur de poser des conditions au mariage ni d'en supprimer certaines ${ }^{6}$. Par ailleurs, autoriser le mariage des personnes de même sexe n'atteint pas la liberté des hétérosexuels de se marier.

Le Conseil affirme en outre que la loi ouvrant le mariage aux couples homosexuels n'est pas contraire au principe d'égalité:

[...] si, en règle générale, ce principe impose de traiter de la même façon des personnes qui se trouvent dans la même situation, il n'en résulte pas pour autant qu'il oblige à traiter différemment des personnes se trouvant dans des situations différentes ${ }^{69}$.

Le traitement identique de situations distinctes est donc possible. Or, « il n'appartient pas au Conseil constitutionnel de substituer son appréciation à celle du législateur sur la prise en compte en matière de mariage, de cette différence de situation ${ }^{70}$. La même formule est reprise concernant l'ouverture de l'adoption aux couples homosexuels ${ }^{71}$. Fréquente en droit des personnes et de la famille $\mathrm{f}^{72}$, elle révèle une autolimitation du contrôle du Conseil. Certains auteurs ont cru y déceler le souhait de ne pas s'immiscer dans les «débats de société » ${ }^{73}$. Cette justification est d'ailleurs donnée par le commentaire de la décision ${ }^{74}$. Il semble pourtant, comme l'ont relevé François Chénedé et Pascale Deumier, que l'étendue du contrôle dépende plutôt du contenu de la prétention des requérants: «droit à » ou «liberté de», revendication d'un statut ou protection d'un état ${ }^{75}$. Le contrôle du Conseil n'a pas pour finalité d'accéder aux revendications de nouveaux droits ni, parallèlement, d'empêcher le législateur d'ouvrir certains droits plus largement dès lors que, dans les deux cas, aucune atteinte aux libertés et aux droits fondamentaux d'autres personnes ne peut être relevée. Comme le précise le Conseil, autoriser le mariage des couples homosexuels est sans incidence sur les «droits acquis nés de mariages antérieurs » par des couples hétérosexuels ${ }^{76}$.

De cette autolimitation du contrôle du Conseil résulte une conception étroite des droits et libertés invoqués en droit de la famille. Tel est notamment le cas du droit au respect de la vie privée, lequel permet seulement de protéger les individus contre des immixtions dans leur sphère d'intimité, mais ne peut pas être utilement invoqué pour obtenir un droit, notamment l'acquisition de la nationalité française. La décision $n^{\circ}$ 2013-354 QPC insiste ainsi sur le fait que «la contestation de nationalité d'une personne ne met pas en cause son droit au respect de la vie privée ${ }^{77}$. Déjà, les décisions n 2012-227 QPC et $n^{\circ}$ 2012-264 QPC avaient affirmé «que ni le respect de la vie privée ni aucune autre exigence constitutionnelle n'impose que le conjoint d'une personne de nationalité française puisse acquérir la nationalité française à ce titre $»^{78}$.

\section{B. Le droit de l'environnement}

Trois décisions rendues par le Conseil sont relatives au principe de participation du public prévu par l'article 7 de la Charte de l'environnement. Ces décisions, qui ne portent que sur le seul champ d'application des décisions soumises à participation, s'inscrivent dans la continuité de la jurisprudence du Conseil sur cette question.

La loi du 27 décembre 2012 et l'ordonnance du 5 août 2013 sont venues élargir le champ d'application des décisions devant faire l'objet de la participation ${ }^{79}$. Jusqu'alors, aucune disposition législative ne prévoyait la participation pour les décisions individuelles des autorités publiques qui, pourtant, auraient une incidence directe et significative

67. Reconnue comme une liberté constitutionnellement protégée depuis 1993 sur le fondement de la liberté individuelle (CC, déc. n $93-325$ DC du 13 août 1993, Loi relative à la maîtrise de l'immigration et aux conditions d'entrée, d'accueil et de séjour des étrangers en France, cons. 107), elle est rattachée depuis 2003 à la liberté personnelle (CC, déc. n² 2003-484 DC du 20 novembre 2003, Loi relative à la maîtrise de l'immigration, au séjour des étrangers en France et à la nationalité, cons. 94).

68. CC, déc. $n^{\circ}$ 2010-92 QPC du 28 janvier 2011, $M^{m e}$ Corinne C. et autre, cons. 7: «La liberté du mariage ne restreint pas la compétence que le législateur tient de l'article 34 de la Constitution pour fixer les conditions du mariage dès lors que, dans l'exercice de cette compétence, il ne prive pas de garanties légales des exigences de caractère constitutionnel».

69. CC, déc. nº 2013-669 DC, cons. 15 .

70. Ibid., cons. 22 .

71. Ibid., cons. 49 .

72. CC, déc. nº 2012-248 QPC du 16 mai 2012, M. Mathieu E., cons. 8; CC, déc. nº 2011-173 QPC du 30 septembre 2011, M. Louis C. et autres, cons. 6; CC, déc. n² 2010-92 QPC, cons. 9; CC, déc. n² 2010-39 QPC, cons. 9.

73. J.-R. Binet, "Conformité de la loi ouvrant le mariage aux couples de personnes de même sexe à la Constitution", Droit de la famille, $\mathrm{n}^{\circ} 7$, 2013, comm. n 98 ; P. Delvolvé, «Constitution et société», Revue française de droit administratif, n 5, 2013, p. 923-926.

74. Commentaire aux Cahiers du Conseil constitutionnel, p. 19 (disponible en ligne: http://www.conseil-constitutionnel.fr/conseil-constitutionnel/ root/bank/download/2013669DCccc_669dc.pdf): «la jurisprudence du Conseil constitutionnel est constante pour souligner la compétence du Parlement sur ces sujets de société».

75. F. Chénedé, P. Deumier, «L'œuvre du Parlement, la part du Conseil constitutionnel en droit des personnes et de la famille», Les nouveaux cahiers $d u$ Conseil constitutionnel, n 39, 2013, p. 7-18; F. Chénedé, «Un autre regard sur la décision du Conseil constitutionnel relative au "mariage pour tous" ", Constitutions, $\mathrm{n}^{\circ} 4,2013, \mathrm{p} .555-563$.

76. CC, déc. $\mathrm{n}^{\circ}$ 2013-669 DC, cons. 23.

77. CC, déc. $\mathrm{n}^{\circ}$ 2013-354 QPC, cons. 10

78. CC, déc. nº 2012-227 QPC du 30 mars 2012, M. Omar S., cons. 8; CC, déc. nº 2012-264 QPC du 13 juillet 2012, M. Saïd K., cons. 6.

79. La loi $\mathrm{n}^{\circ} 2010-788 \mathrm{du} 12$ juillet 2010 portant engagement national pour l'environnement ne prévoyait la participation que pour les seules « décisions réglementaires de l'État et de ses établissements publics» qui avaient une «incidence directe et significative sur l'environnement». 
sur l'environnement. L'élargissement est double puisque sont désormais soumises à la participation du public les décisions individuelles ${ }^{80}$, et pour les «décisions, autres que les décisions individuelles, des autorités de l'État ${ }^{81}$, l'exigence d'une incidence directe et significative sur l'environnement disparaît. Toutefois, cette dernière condition a été réintroduite par l'ordonnance du 5 août 2013 pour les seules décisions individuelles ${ }^{82}$.

Malgré le caractère "peu lisible ${ }^{8_{3}}$ de la jurisprudence du Conseil sur cette question, tout porte à croire que dans tous les cas et malgré l'intervention de la loi de 2012, le Conseil est amené à contrôler le caractère direct et significatif sur l'environnement de la décision ${ }^{84}$ pour déterminer si la décision en question doit être ou non soumise à la participation du public. Le contrôle de l'existence d' « une incidence significative sur l'environnement $»^{85}$ des autorisations de travaux de recherche minière est justifié, dans la mesure où il s'agit d'une décision individuelle entrant dans le champ de l'ordonnance d'août 2013 précitée ${ }^{86}$. Toutefois, de manière plus surprenante, le Conseil opère le même contrôle concernant des décisions réglementaires. Ainsi, le décret en Conseil d'État auquel le législateur renvoie le soin de fixer la quantité minimale de matériaux en bois dans certaines constructions nouvelles est bien, selon le Conseil, une décision publique, mais «l'exigence de telles normes techniques n'est, en elle-même, susceptible de n'avoir qu'une incidence indirecte sur l'environnement ${ }^{87}$. En effet,

[...] le lien entre l'introduction du bois dans les constructions nouvelles et le maintien et le développement de la forêt est à la fois indirect et incertain. En particulier, rien ne garantit que l'augmentation de la consommation de bois conduise à l'augmentation de la surface des forêts ${ }^{88}$.

Cette solution n'allait pas de soi car, dans une précédente décision, le Conseil avait considéré que les projets de règles et prescriptions techniques, que doivent respecter les installations classées pour la protection de l'environnement soumises à autorisation, constituent des décisions publiques ayant une incidence sur l'environnement ${ }^{89}$.

Dans sa décision nº 2013-346 QPC, le Conseil a déclaré conformes à la Constitution des dispositions du Code de l'environnement issues de la loi du 13 juillet 2011 relatives à l'interdiction de la fracturation hydraulique pour l'exploration et l'exploitation des hydrocarbures et à l'abrogation des permis de recherches dans ce domaine. Parmi les nombreux griefs des requérants ${ }^{90}$, était invoqué le principe de précaution consacré par l'article 5 de la Charte de l'environnement. Pour le Conseil, dans la mesure où l'interdiction est "pérenne», et non pas provisoire comme il est mentionné à l'article 5, «les dispositions considérées n’ont donc pas été prises en application du principe de précaution ${ }^{91}$, mais en vertu du principe de prévention. La solution retenue par le Conseil n'était cependant pas évidente, au regard du rattachement incertain de cette interdiction au principe de prévention ${ }^{92}$.

Si la Charte a été invoquée dans chacune de ces décisions portant sur l'environnement, c'est au regard d'autres dispositions constitutionnelles que le Conseil a rendu deux décisions d'inconstitutionnalité. En effet, dans sa décision

80. Art. L. 120-1-1 du Code de l'environnement, créé par l'ordonnance 2013-714 du 5 août 2013.

81. Art. L. 120-1 du Code de l'environnement.

82. Art. L. 120-1-1 du Code de l'environnement: «le présent article définit les conditions et limites dans lesquelles le principe de participation du public prévu à l'article 7 de la Charte de l'environnement est applicable aux décisions individuelles des autorités publiques ayant une incidence sur l'environnement [...]. Ne sont pas regardées comme ayant une incidence sur l'environnement les décisions qui ont sur ce dernier un effet indirect ou non significatif» (nous soulignons).

83. Voir K. Foucher, «Un an de Charte de l'environnement au Conseil constitutionnel (novembre 2012 à octobre 2013 ): les incertitudes d'une jurisprudence en voie de construction", Constitutions, $\mathrm{n}^{\circ}$ 4, 2013, p. 606-619.

84. À propos de CC, déc. nº 2013-316 QPC du 24 mai 2013, SCI Pascal et autre, voir N. Foulquier, «Le domaine public maritime naturel. La soi-disant évidence de la nature", L'actualité juridique. Droit administratif, nº 39, 2013, p. 2260-2263; toutefois, comme le précise K. Foucher, il est arrivé que le Conseil considère qu'une décision ayant un caractère indirect mais significatif sur l'environnement entre dans le champ de l'article 7 ( «Un an de Charte de l'environnement...»).

85. CC, déc. $\mathrm{n}^{\circ}$ 2013-308 QPC du 26 avril 2013, Association «Ensemble pour la planète» [Nouvelle-Calédonie-Autorisations de travaux de recherches minières], cons. 11.

86. Il faut toutefois se référer au commentaire aux Cahiers du Conseil constitutionnel, p. 14 (disponible en ligne: http://www.conseil-constitutionnel. fr/conseil-constitutionnel/root/bank/download/2013308QPCccc_308qpc.pdf) pour comprendre que «le Conseil est parvenu à cette solution en apportant deux précisions dans son considérant 11. Il importe en effet de tenir compte: d'une part de la nature des substances minérales susceptibles d'être recherchées [...]; d'autre part des techniques mises en œuvre en l'état».

87. CC, déc. nº 2013-317 QPC du 24 mai 2013, Syndicat français de l'industrie cimentière et autre, cons. 7 (nous soulignons).

88. Commentaire aux Cahiers du Conseil constitutionnel, p. 8 (disponible en ligne: http://www.conseil-constitutionnel.fr/conseil-constitutionnel/ root/bank/download/2013317QPCccc_317qpc.pdf).

89. F.-G. Trébulle, «Droit de l'environnement septembre 2012 - octobre 2013», Dalloz, 2014, p. 106-107, à propos de CC, déc. nº 2012-262 QPC du 13 juillet 2012, Association France Nature Environnement.

90. Voir L. Fonbaustier, «Industrie des hydrocarbures: de l'eau dans le gaz? À propos de la décision du Conseil constitutionnel du 11 octobre 2013 », La semaine juridique, éd. $G$, $\mathrm{n}^{\circ}$ 44, 28 octobre 2013, p. 1993-1995.

91. P. Billet, "QPC "Gaz de schiste": validation de la loi "Jacob”", Environnement, no 1, 2014, p. 25.

92. Voir L. Fonbaustier, «Industrie des hydrocarbures...», p. 1995: «Le raisonnement des juges est ici elliptique, dans la mesure où ils semblent affirmer tout à la fois [...] que la loi de 2011 met en œuvre le principe de prévention (on se situerait donc en terrain de risques connus...) mais que cette situation semble justifiée en l'état des connaissances et des techniques (cons. 10). Ou bien le Conseil aurait dû limiter sa référence aux techniques (sous-entendu, d'exploration et / ou d'exploitation), ou bien cette formulation révèle une gêne en ce qui concerne l'applicabilité, en l'espèce, du principe de précaution... Finalement, c'est plutôt le fait que les techniques ici employées paraissent suffisamment connues dans leurs effets (point qui fait semble-t-il actuellement débat) qui explique que le législateur ait entendu se placer sur le terrain de la prévention et non sur celui de la précaution». 
$\mathrm{n}^{\circ}$ 2013-317 QPC ${ }^{93}$, le Conseil censure le paragraphe V de l'article L. 224-1 du Code de l'environnement, au motif qu'il est contraire à la liberté d'entreprendre, et non au principe de participation ${ }^{94}$. Dans sa décision $\mathrm{n}^{\circ}{ }^{2013-}$ 666 DC, le Conseil a censuré plusieurs dispositions de la loi visant à préparer la transition vers un système énergétique sobre, qu'il a déclarées contraires au principe d'égalité devant les charges publiques. C'est le dispositif instaurant un mécanisme de bonus-malus pour les consommations en matière d'électricité qui est l'objet de cette censure ${ }^{95}$. Le Conseil a suivi un raisonnement analogue à celui de sa décision $n^{\circ}$ 2009-599 DC qui a censuré le dispositif « taxe carbone ${ }^{96}$, pour censurer l'article 2 ainsi que l'ensemble du titre $I^{\text {er }}$ de la loi en question. En effet, le Conseil relève d'abord que ces dispositions, en ce qu'elles ne s'appliquent qu'aux seules consommations domestiques des particuliers, méconnaissent le principe d'égalité. Ensuite, il considère que:

[...] ces dispositions n'assurent pas le respect de l'égalité devant les charges publiques, d'une part, entre les consommateurs qui résident dans ces immeubles collectifs et, d'autre part, avec les consommateurs domestiques demeurant dans un site de consommation résidentiel individuel ${ }^{97}$.

Cette décision, qui était somme toute assez prévisible $^{98}$, n'en est pas moins contestable. Comme l'a souligné Martine Lombard, on peut regretter que le Conseil ne se soit pas davantage inspiré de «la conception plus pragmatique de l'égalité que semble retenir le juge européen " ${ }^{99}$, et qu'il n'ait pas pris en compte le fait que ce dispositif était considéré comme un "premier acte ${ }^{100}$. Surtout, l'auteur relève qu'il faut espérer que «les conséquences de cette récente décision du Conseil constitutionnel ne seront pas identiques à celles de sa précédente décision sur la taxe carbone», mettant ainsi en garde contre le risque $\mathrm{d}^{\prime}$ «immobilisme» ${ }^{101}$. S'agissant de cette même décision, il faut relever que sans surprise ${ }^{102}$, le Conseil a déclaré conforme à l'article 6 de la Charte les articles 26 et 29 de la loi qui visaient à faciliter l'implantation d'éoliennes dans les communes d'outre-mer et à supprimer la règle dite des «cinq mâts» ${ }^{103}$.

\section{La protection du droit de propriété}

Plusieurs décisions viennent en 2013 apporter des précisions sur la notion de bien susceptible d'être protégé. Ainsi,

[...] les autorisations de recherche minière accordées dans des périmètres définis et pour une durée limitée par l'autorité administrative ne sauraient être assimilées à des biens objets pour leurs titulaires d'un droit de propriété [... $]^{104}$.

Si cette décision s'inscrit dans la continuité de la jurisprudence du Conseil ${ }^{105}$, elle s'avère discutable, puisque

La définition des biens que retient le Conseil demeure particulièrement restrictive, singulièrement au regard de celle retenue par la Cour européenne des droits de l'homme ${ }^{106}$.

En outre, dans une décision très critiquée, le Conseil a précisé que ne peuvent faire l'objet d'une propriété privée «les espaces couverts, même épisodiquement, par les flots ${ }^{107}$, puisqu'en

[...] prévoyant que cette limite [entre le domaine public maritime naturel et les propriétés privées] est fixée en fonction de tout ce que la mer "couvre et découvre jusqu'où les plus hautes mers peuvent s'étendre en l'absence de perturbations météorologiques exceptionnelles», le législateur a confirmé un critère physique objectif indépendant de la volonté de la puissance publique ${ }^{108}$.

Or, comme l'a souligné Hélène Hoepffner,

Le mouvement de la mer n'est pas en lui-même attributif de propriété: certes, lorsque la mer monte, le domaine public maritime s'étend; mais c'est parce que la loi a fait de la mer une dépendance domaniale, qu'elle a lié la domanialité publique au mouvement des flots en définissant le rivage

93. CC, déc. $\mathrm{n}^{\circ}$ 2013-317 QPC, cons. 10.

94. Dans cette décision, qui constitue l'une des rares censures au regard de la liberté d'entreprendre, le Conseil cite l'article 4 de la DDHC dans sa totalité, précisant ainsi «de manière très ferme les conditions dans lesquelles une loi intervenant au nom de la protection de l'environnement doit se concilier avec la liberté d'entreprendre»; B. Julié, «Une volée de bois vert? Protection de l'environnement et liberté d'entreprendre ", Gazette du palais, ${ }^{\text {er }}$ août 2013, p. 13.

95. CC, déc. $\mathrm{n}^{\circ}$ 2013-666 DC, Loi visant à préparer la transition vers un système énergétique sobre et portant diverses dispositions sur la tarification de l'eau et sur les éoliennes, cons. 18.

96. Ibid., cons. 9. Voir CC, déc. nº 2009-599 DC du 29 décembre 2009, Loi de finances pour 2010.

97. Ibid., cons. 17

98. Sur ce point, voir S. Nicinski, «Les économies de consommation d’énergie devant le Conseil constitutionnel», Revue juridique de l'économie publique, $\mathrm{n}^{\circ} 713,2013$, p. 23-24.

99. M. Lombard, "Selon le Conseil constitutionnel, intervenir n'est pas choisir», in "Actualités du droit de la concurrence et de la régulation", L'actualité juridique. Droit administratif, nº 22, 2013, p. 1264.

100. Assemblée nationale, $14^{\mathrm{e}}$ législature, proposition de loi n 150, p. 3 ; cité par M. Lombard, «Selon le Conseil... », p. 1264.

101. M. Lombard, «Selon le Conseil...», p. 1264.

102. Commentaire aux Cahiers du Conseil constitutionnel, p. 22 (disponible à l'adresse: http://www.conseil-constitutionnel.fr/conseil-constitutionnel/ root/bank/download/2013666DCccc_666dc.pdf).

103. CC, déc. nº 2013-666 DC, cons. 40.

104. CC, déc. $\mathrm{n}^{\circ}$ 2013-346 QPC, cons. 17

105. Cette solution s'inscrit dans le cadre d'une décision rendue à propos d'autorisations d'exploitation de services de transports publics; voir CC, déc. n 82-150 DC du 30 décembre 1982, Loi d'orientation des transports intérieurs.

106. F.-G. Trébulle, «Droit de l'environnement...», p. 106

107. CC déc. $\mathrm{n}^{\circ}$ 2013-316 QPC, cons. 6.

108. Ibid. 
que celui-ci ne peut faire l'objet d'une appropriation privée ${ }^{109}$.

Classiquement, il convient de rappeler que le droit de propriété est protégé par deux dispositions constitutionnelles, l'article 17 de la DDHC sanctionnant la privation ou dénaturation du droit de propriété et, l'article 2 concernant les limitations ${ }^{110}$. La décision $\mathrm{n}^{\circ}$ 2013-325 QPC apporte une précision sur le champ d'application de l'article 17. En effet, n'entre pas dans ce champ le transfert de propriété résultant de l'exercice du « droit de délaissement au profit des propriétaires de terrains, bâtis ou non bâtis, classés en emplacements réservés par un plan d'urbanisme » dans la mesure où il s'agit d'une « réquisition d'achat à l'initiative des propriétaires de ces terrains ${ }^{111}$, et non de la puissance publique. Cette situation entre toutefois dans le champ de l'article 2, même si, pour le Conseil, cette disposition est conforme à la Constitution.

On observe que le Conseil n'a rendu cette année qu'une seule décision de censure sur le fondement du droit de propriété ${ }^{12}$, à propos d'une disposition du Code rural et de la pêche maritime applicable en matière de baux ruraux ${ }^{113}$. Cette disposition prévoit que les sommes perçues à l'occasion de "pas-de-porte» sont sujettes à répétition et «majorées d'un intérêt calculé à compter de leur versement et égal au taux pratiqué par la Caisse régionale de crédit agricole pour les prêts à moyen terme ${ }^{114}$. Le Conseil, qui s'était saisi d'office de ce grief, a censuré la deuxième partie de cette disposition («et égal au taux pratiqué par la Caisse régionale de crédit agricole pour les prêts à moyen terme»), au motif «qu'en s'abstenant de fixer ou d'habiliter le pouvoir réglementaire à fixer les modalités selon lesquelles le taux prévu par les dispositions contestées est déterminé et rendu public, le législateur a méconnu l'étendue de la compétence qu'il tient de l'article 34 de la Constitution ${ }^{115}$, et "que l'absence de détermination des modalités de calcul du taux d'intérêt applicable à une créance affecte par elle-même le montant des sommes allouées et, par suite, le droit de propriété tant du créancier que du débiteur ${ }^{116}$. Une autre censure eût été envisageable en matière d'expropriation ${ }^{117}$, domaine qui, cette année encore, a fait l'objet de plusieurs décisions. À cet égard, plusieurs auteurs relèvent l'insuffisance de motivation de certaines décisions, à l'image de René Hostiou qui parle d' "indigence de la motivation ${ }^{118}$ à propos de la décision n ${ }^{\circ}$ 2012-292 QPC portant sur le droit de rétrocession en cas d'expropriation pour cause d'utilité publique ${ }^{119}$. En effet, le Conseil a jugé que la seule réquisition d'une nouvelle déclaration d'utilité publique, qui «permet à elle-seule de faire obstacle à une demande de rétrocession formée par l'ancien propriétaire ou ses ayants droit $»^{120}$, n'était pas contraire au droit de propriété. Dès lors,

[...] sous la seule condition de requérir une nouvelle DUP [déclaration d'utilité publique], l'expropriant est assuré de pouvoir continuer à disposer ad libitum du bien exproprié, qu'il ait simplement pris du retard dans la réalisation de l'opération prévue sans avoir pour autant renoncé à celle-ci ou qu'il ait finalement changé d'avis et qu'il envisage de substituer à l'affectation initialement prévue une affectation différente ${ }^{121}$.

\section{La libre administration des collectivités territoriales}

En 2013, les références à la libre administration des collectivités territoriales ont été riches et surtout variées ${ }^{122}$. Pour autant, le sens de la jurisprudence du Conseil relative à ce principe que la Constitution garantit ${ }^{123}$ est univoque: en dépit de son organisation décentralisée, la République

109. H. Hoepffner, «Les "transferts naturels de propriété": forme d'expropriation indirecte sans indemnisation?", Les nouveaux cahiers du Conseil constitutionnel, $\mathrm{n}^{\circ} 41,2013$, p. 296; voir également G. Eveillard, "La constitutionnalité de la consistance du domaine public maritime», Droit administratif, $n^{\circ} 10$, octobre 2013 , comm. $\mathrm{n}^{\circ} 70 ; \mathrm{N}$. Foulquier, «Le domaine public maritime naturel...».

110. Voir par exemple, CC déc. nº 2013-316 QPC, cons. 3.

111. CC, déc. no 2013-325 QPC du 21 juin 2013, M. Jean-Sébastien C., cons. 5.

112. On remarquera toutefois que certaines décisions ont été censurées ou ont fait l'objet de réserves, mais sur d'autres fondements que le droit de propriété, par exemple: CC, déc. n 2012-298 QPC; CC, déc. n²013-679 DC du 4 décembre 2013, Loi relative à la lutte contre la fraude fiscale et la grande délinquance économique et financière; CC, déc. n²013-327 QPC du 21 juin 2013, SA Assistance Sécurité et Gardiennage.

113. CC, déc. n 2013-343 QPC.

114. Art. L. 411-74 du Code rural et de la pêche maritime.

115. CC, déc. nº 2013-343 QPC, cons. 6.

116. Ibid., cons. 7; cette décision s'inscrit dans le cadre d'une précédente décision du Conseil: CC, déc. n²010-607 DC du 10 juin 2010, Loi relative à l'entrepreneur individuel à responsabilité limitée, cons. 9.

117. CC, déc. n² 2013-342 QPC du 20 septembre 2013, SCI de la Perrière Neuve et autre. Les requérants n'ont en effet pas soulevé les bonnes dispositions. Voir par exemple S. Gilbert, «La constitutionnalité des garanties d'indemnisation de l'emphytéote en cas d'expropriation », L'actualité juridique. Droit administratif, $\mathrm{n}^{\circ} 43,2013$, p. 2498.

118. Sur ce point, voir R. Hostiou, «La mise en échec du droit de rétrocession par la réquisition d'une nouvelle déclaration d'utilité publique: conformité à la Constitution. Note sous Conseil constitutionnel, 15 février 2013, $M^{\text {me }}$ Suzanne Prat-Audemar, décision n ${ }^{\circ} 2012-292$ QPC», Revue française de droit administratif, $\mathrm{n}^{\circ} 2,2013$, p. 263.

119. CC, déc. $\mathrm{n}^{\mathrm{o}}$ 2012-292 QPC du 15 février 2013, $M^{\text {me }}$ Suzanne P.-A.; on pourrait également mentionner CC, déc. $\mathrm{n}^{\circ}$ 2013-338/339 QPC.

120. Ibid., cons. 6 .

121. R. Hostiou, «La mise en échec... », p. 262.

122. À l'exception de la liberté contractuelle des collectivités territoriales, les conditions du respect du principe de libre administration des collectivités territoriales ont toutes été rappelées par les décisions du Conseil constitutionnel en 2013 : l'existence d'un conseil élu, l'attribution de compétences effectives, la garantie de l'autonomie financière.

123. CC, déc. nº 2010-12 QPC, cons. 4. 
demeure jacobine ${ }^{124}$. Au fil del'année, le juge constitutionnel s'appuie sur les articles 34, 72 et 72-2, tantôt pour conférer au législateur le soin d'encadrer la libre administration des collectivités territoriales (1), tantôt pour lui permettre d'y porter légitimement atteinte (2). Dès lors, quand il en vient à censurer le législateur au nom de la libre administration des collectivités territoriales, l'occurrence mérite d'être relevée même si la décision a une portée limitée (3).

\section{L'encadrement de la libre administration des collectivités territoriales par le législateur}

Plusieurs décisions confirment que le principe de libre administration des collectivités territoriales fonde, tout d'abord, la compétence du législateur à encadrer la liberté des collectivités territoriales autres que l'État. Le Conseil rappelle que selon les articles 72 et 72-2 de la Constitution, les collectivités territoriales «s'administrent librement par des conseils élus » et «bénéficient de ressources dont elles peuvent disposer librement ", mais il considère surtout que ces dispositions ne valent que "dans les conditions prévues par la loi». Ainsi, le grief ne pouvait qu'être écarté lors de l'examen de l'article 24 de la loi du 15 avril 2013 relative à la transition énergétique. Celui-ci supprime les zones de développement de l'éolien (ZDE) qui permettaient aux communes et aux établissements de coopération intercommunale (EPCI) de favoriser l'installation d'éoliennes sur leur territoire, laissant place au seul schéma régional d'implantation de l'éolien, déterminé par le préfet de région, comme cadre administratif. Si les communes et les EPCI perdent un levier pour augmenter indirectement leurs ressources, le Conseil considère, par la décision $n^{\circ}$ 2013-666 DC, que l'article contesté ne porte pas atteinte à la libre administration des collectivités territoriales car il n'affecte pas en soi les recettes des communes, ne porte atteinte ni aux compétences de celles-ci, ni à celles des EPCI, et n'a pas pour effet d'instaurer une « quasi-tutelle» de la région sur les communes ${ }^{125}$.

Le législateur peut également réguler directement les ressources fiscales des collectivités territoriales sans pour autant attenter au principe de libre administration des collectivités territoriales comme l'attestent quatre décisions du Conseil en 2013. La création d'un prélèvement sur les recettes fiscales, à l'encontre des communes qui n'ont pas atteint le taux de logements locatifs sociaux exigé, n'a pas pour effet de réduire les ressources globales des communes, ni de diminuer leurs ressources fiscales au point de porter atteinte à leur libre administration ${ }^{126}$. La détermination de tarifs maximaux applicables à la taxe locale sur la publicité extérieure dans certaines communes, par l'article L. 2333-16 du Code général des collectivités territoriales issu de la loi de modernisation de l'économie de 2008 , ne réduit pas leurs ressources propres dans des proportions telles que serait méconnue l'autonomie financière des communes ${ }^{127}$. Le principe constitutionnel de libre administration des collectivités territoriales est donc respecté. De même, le dispositif de compensation financière des pertes de recettes fiscales de l'État, prévu par la loi de finances de 2010 en contrepartie du transfert du produit de la taxe sur les surfaces commerciales (TASCOM) du budget de l'État à celui des communes et des EPCI à fiscalité propre, est conforme au principe constitutionnel. En effet, même si cette règle peut conduire à la diminution de certains budgets communaux, le législateur a entendu, par ce dispositif, renforcer l'autonomie financière des communes ${ }^{128}$. Enfin, par la loi de finances pour 2014, le législateur a pu, d'une part, instaurer un plafonnement des prélèvements à destination $\mathrm{du}$ fond de solidarité en faveur des départements, et du fond de mutualisation des titres onéreux, sans pour autant restreindre les ressources des départements au point de dénaturer le principe de libre administration, et, d'autre part, subordonner l'aide d'un fond de soutien destinée aux collectivités territoriales à la conclusion d'une transaction entre une collectivité intéressée et un établissement financier prêteur, sans violation dudit principe ${ }^{129}$.

\section{Les atteintes légitimes à la libre administration des collectivités territoriales}

Les articles $34^{130}$ et $72^{131}$ de la Constitution autorisent ensuite le législateur à affecter la libre administration des collectivités territoriales, notamment en prévoyant l'intervention du préfet aux dépens des autorités locales, quand l'intérêt général le justifie et que les atteintes sont proportionnées à l'objectif poursuivi. Ainsi en 2013, le Conseil admet que la décision du représentant de l'État se substitue à celles des exécutifs territoriaux en matière de réalisation de l'objectif de mixité sociale et de coopération intercommunale ${ }^{132}$.

124. Voir J.-H. Stahl, «Le principe de libre administration a-t-il une portée normative? », Les nouveaux cahiers du Conseil constitutionnel, $\mathrm{n}^{\circ}$ 42, 2014, p. 32 : «Le substrat jacobin demeure, la Constitution en porte la marque : la République est une et indivisible, même si elle reconnaît, depuis plus d'un siècle, les libertés locales et si, depuis 2003 , "son organisation est décentralisée" ".

125. CC, déc. $\mathrm{n}^{\circ}$ 2013-666 DC, cons. 35 .

126. CC, déc. $\mathrm{n}^{\circ}$ 2012-66o DC du 17 janvier 2013, Loi relative à la mobilisation du foncier public en faveur du logement et au renforcement des obligations de production de logement social, cons. 20 et 21.

127. CC, déc. $\mathrm{n}^{\circ}$ 2013-305/306/307 QPC, cons. 13.

128. CC, déc. no 2013-355 QPC du 22 novembre 2013, Communauté de communes du Val de Sèvre, cons. 5 et 6.

129. CC, déc. no 2013-685 DC du 29 décembre 2013, Loi de finances pour 2014, cons. 67 et 75.

130. Art. 34, al. 3 de la Constitution: «La loi détermine les principes fondamentaux: [...] de la libre administration des collectivités territoriales, de leurs compétences et de leurs ressources [...]».

131. Art. 72, al. 6 de la Constitution: «Dans les collectivités territoriales de la République, le représentant de l'État, représentant de chacun des membres du Gouvernement, a la charge des intérêts nationaux, du contrôle administratif et du respect des lois ».

132. Voir G. Le Chatelier, «Le Conseil constitutionnel valide les pouvoirs exorbitants du préfet en matière d'intercommunalité», L'actualité juridique. Collectivités territoriales, $\mathrm{n}^{\circ} 7,2013$, p. 344-346. 
En premier lieu, dans le but de mettre en œuvre l'objectif de mixité sociale et d'accroissement de la production de logements locatifs sociaux, l'article 16 de la loi du 18 janvier 2013 relative à la mobilisation du foncier public en faveur du logement et au renforcement des obligations de production de logement social permet au préfet de prononcer un constat de carence à l'encontre des communes qui n'ont pas atteint le taux fixé par la loi. Selon la décision $n^{\circ}$ 2012-66o DC, si cette procédure a pour conséquence d'imposer de nouvelles contraintes aux communes et d'alourdir les prélèvements sur les ressources fiscales de celles concernées par le constat de carence, l'atteinte portée à la libre administration des collectivités territoriales n'est pas d'une gravité suffisante pour que le principe constitutionnel soit méconnu ${ }^{133}$. En outre, le droit de préemption du préfet, prévu à l'article L. 210-1 du Code de l'urbanisme et applicable aux communes ayant fait l'objet d'un constat de carence, dans le cadre de la procédure précitée, a pour effet de permettre au représentant de l'État de se substituer à la défaillance de la commune afin de ne pas compromettre le fonctionnement des services publics et l'application des lois. Dès lors, contrairement à ce que mentionne le commentaire de la décision $\mathrm{n}^{0}$ 2013-309 $\mathrm{QPC}^{134}$, le droit de préemption du préfet porte une atteinte à la libre administration des collectivités territoriales, mais celle-ci ne revêt pas un caractère disproportionné par rapport à l'objectif poursuivi ${ }^{135}$.

En second lieu, afin de favoriser l'intercommunalité, l'article 60 de la loi du 16 décembre 2010 portant réforme des collectivités territoriales confère au préfet le pouvoir de fusionner des EPCI ou d'en modifier le périmètre. Par les décisions no 2013-303 QPC et n² 2013-315 QPC, le Conseil constitutionnel admet que la mise en œuvre de ces prérogatives affecte la libre administration des collectivités territoriales dans la mesure où elle a pour conséquence d'imposer à des communes de faire partie d'un EPCI, même quand celles-ci souhaitent appartenir à un autre établissement du même type ${ }^{136}$. Pour autant, parce que le législateur a entendu favoriser «la rationalisation de la carte de l'intercommunalité », que la procédure contraignante n'est applicable que jusqu'au $1^{\text {er }}$ juin 2013 , et que tout maire d'une commune concernée peut être entendu par la commission départementale de la coopération intercommunale, les limitations à la libre administration des communes sont justifiées par l'intérêt général et ne méconnaissent pas le principe constitutionnel de libre administration des collectivités territoriales ${ }^{137}$. De même, par la décision n²013-304 QPC, le Conseil déclare que l'article L. 5211-19 du Code général des collectivités territoriales, qui subordonne le retrait, prononcé par arrêté du préfet, d'une commune d'un EPCI à l'accord de l'organe délibérant de l'établissement et à une majorité qualifiée de conseil municipaux des communes concernées, est conforme à la Constitution. Si les règles relatives au retrait d'une commune d'un EPCI affectent la libre administration de celle-ci, le fonctionnement et la stabilité de ces établissements et la cohérence de la coopération communale constituent des buts d'intérêt général suffisants pour limiter la libre administration des communes ${ }^{138}$. Au fil des ans, le Conseil précise le contenu du principe constitutionnel sans pour autant renier une conception stato-centrée de la libre administration des collectivités territoriales, dont le législateur demeure le principal ordonnateur et le représentant de l'État, l'indubitable tuteur.

\section{Le législateur censuré au nom de la libre administration des collectivités territoriales}

À l'occasion de l'examen de la règle d'inéligibilité du directeur de cabinet du président du conseil régional aux élections municipales prévue à l'article L. 231 du Code électoral, le Conseil a rappelé, dans la décision n 2013-326 QPC, qu'il ne dispose pas d'un pouvoir général d'appréciation de même nature que celui du Parlement. Il a donc exercé, en l'espèce, un contrôle minimum pour considérer que les dispositions contestées n'opéraient pas une conciliation «manifestement déséquilibrée» entre les exigences constitutionnelles, parmi lesquelles figurait le principe de libre administration des collectivités territoriales ${ }^{139}$. Malgré cette prudence à l'égard de l'œuvre du législateur en matière électorale, le Conseil a censuré l'article 15 de la loi relative à l'élection. Les nouveaux conseillers départementaux étant élus par binôme de candidats, il n'était pas prévu d'organiser d'élection partielle tant que les deux sièges du binôme n'étaient pas vacants. Le Conseil a donc considéré, par la décision n 2013-667 DC, que la vacance de plusieurs sièges de conseillers départementaux pouvait durer six ans et que cela affectait le fonctionnement du conseil départemental dans des conditions remettant en cause le principe selon lequel les collectivités territoriales s'administrent librement par des conseils élus ${ }^{140}$.

133. CC, déc. n 2012-66o DC du 17 janvier 2013, Loi relative à la mobilisation du foncier public en faveur du logement et au renforcement des obligations de production de logement social, cons. 33 .

134. Commentaire aux Cahiers du Conseil constitutionnel, p. 11 (disponible en ligne: http://www.conseil-constitutionnel.fr/conseil-constitutionnel/ root/bank/download/2013309QPCccc_309qpc.pdf): «Tous ces éléments ont conduit le Conseil constitutionnel à juger que la restriction du pouvoir des communes dont la carence a été constatée ne portait pas atteinte à la libre administration des collectivités territoriales ".

135. CC, déc. nº 2013-309 QPC du 26 avril 2013, SARL SCMC, cons. 5

136. CC, déc. $\mathrm{n}^{\circ}$ 2013-303 QPC, cons. 10; CC, déc. nº 2013-315 QPC, cons. 10.

137. CC, déc. no 2013-303 QPC, cons. 10.

138. CC, déc. n² 2013-304 QPC, cons. 6.

139. CC, déc. $\mathrm{n}^{\circ}$ 2013-326 QPC du 5 juillet 2013, M. Jean-Louis M., cons. 4.

140. CC, déc. $\mathrm{n}^{\circ}$ 2013-667 DC du 16 mai 2013, Loi relative à l'élection des conseillers départementaux, des conseillers municipaux et des conseillers communautaires, et modifiant le calendrier électoral, cons. 21. 


\section{E. Les garanties de l'article 16 de la Déclaration des droits de l'homme et du citoyen (DDHC)}

Nombreuses sont encore cette année les QPC fondées sur une violation de l'article 16 de la DDHC. Le Conseil constitutionnel y rattache en effet l'ensemble des exigences qui garantissent le droit à une procédure juste et équitable (séparation des pouvoirs, impartialité et indépendance des juridictions, droit à un recours effectif, droits de la défense), ainsi que la garantie des droits.

Concernant, tout d'abord, le principe de séparation des pouvoirs, le Conseil a jugé non conformes à la Constitution les dispositions qui imposent la présence du ministre du Budget lors des débats devant les commissions permanentes compétentes en matière de finances de l'Assemblée nationale et du Sénat dans sa décision n ${ }^{0}$ 2013-679 DC ${ }^{141}$. De même, il a précisé, dans la décision no 2012-675 DC, que le principe de séparation des pouvoirs fait obstacle à ce qu'une autorité administrative indépendante puisse formuler à l'égard d'un député ou d'un sénateur une injonction en lien, même indirect, avec l'exercice de son mandat ${ }^{142}$.

Plusieurs décisions ont, ensuite, réaffirmé les exigences d'impartialité et d'indépendance des juridictions. La décision n² 2013-310 QPC concerne plus spécialement la création, par la loi du 11 février 2004, d'une formation disciplinaire distincte du conseil de l'ordre des barreaux dans le ressort de chaque cour d'appel afin de prendre en compte les règles du procès équitable issues de l'article 16 de la DDHC. L'exclusion du barreau de Papeete de cette réforme était contestée par les requérants. Le Conseil affirme au contraire que «le maintien du conseil de l'ordre d'un barreau dans ses attributions disciplinaires n'est pas, en lui-même, contraire aux exigences d'indépendance et d'impartialité de l'organe disciplinaire ${ }^{143}$. Le fait que le législateur ait entendu, en 2004, renforcer l'impartialité et l'indépendance de l'organe disciplinaire n'implique pas, en effet, que la législation antérieure était inconstitutionnelle. Il émet toutefois une réserve d'interprétation selon laquelle les dispositions de la loi ne sauraient permettre au bâtonnier en exercice, ainsi qu'aux anciens bâtonniers ayant engagé la poursuite disciplinaire, de siéger dans la formation disciplinaire du conseil de l'ordre du barreau de Papeete $^{144}$. La décision $n^{\circ}$ 2013-331 QPC, confirme quant à elle l'extension des exigences d'impartialité et d'indépendance aux autorités administratives indépendantes ${ }^{145}$. En l'espèce, le Conseil censure les dispositions des douze premiers alinéas de l'article L. 36-11 du Code des postes et des communications électroniques, "qui n'assurent pas la séparation au sein de l'Autorité entre, d'une part, les fonctions de poursuite et d'instruction des éventuels manquements et, d'autre part, les fonctions de jugement des mêmes manquements ${ }^{146}$.

Concernant le droit à un recours effectif, la question des validations législatives a de nouveau été soumise au contrôle du Conseil en 2013. Une telle intervention du législateur limite l'effectivité du recours puisque des actes administratifs pris sur le fondement d'une décision annulée par le juge pourront produire effet. Le Conseil reprend son attendu de principe, selon lequel la conformité de la validation à la Constitution dépend de l'existence ou non d'un motif d'intérêt général ${ }^{147}$. Si tel était bien le cas concernant l'article 39 de la loi du 16 août 2012 relative aux modalités de recouvrement de la taxe additionnelle à la cotisation sur la valeur ajoutée des entreprises ${ }^{148}$, le Conseil considère au contraire, dans sa décision $n^{\circ} 2012-$ 287 QPC, que :

[...] les motifs financiers invoqués à l'appui de la validation des rémunérations faisant l'objet d'une instance en cours [...], qui portent sur des sommes dont l'importance du montant n'est pas établie, ne peuvent être regardés comme suffisants ${ }^{149}$.

Plusieurs décisions rendues en 2013 rappellent les règles applicables quant au droit à un recours effectif. Le Conseil a ainsi confirmé, dans sa décision n 2013$675 \mathrm{DC}^{150}$, que le silence de la loi concernant l'existence de recours ne porte pas en soi atteinte à l'article $16 \mathrm{de}$ la $\mathrm{DDHC}$, dans la mesure où les règles habituelles ont vocation à s'appliquer. Il a également rappelé en 2013 que le droit à un recours effectif peut être limité, à condition que les restrictions posées soient à la fois motivées par un objectif d'intérêt général et proportionnées à l'objectif poursuivi. Ces deux conditions étant remplies, le Conseil a ainsi validé l'article 414-2 du Code civil relatif aux actions en nullité d'un acte à titre onéreux engagées par les héritiers dans sa décision $n^{\circ}$ 2012-288 QPC ${ }^{151}$. Dans la décision $\mathrm{n}^{\circ}$ 2013-350 QPC ${ }^{152}$, le Conseil a au contraire considéré que l'article 48 de la loi de 1881 sur la protection de la

141. CC, déc. nº 2013-679 DC, cons. 79.

142. CC, déc. $\mathrm{n}^{\circ}$ 2013-675 DC du 9 octobre 2013, Loi organique relative à la transparence de la vie publique, cons. 39.

143. CC, déc. nº 2013-310 QPC du 16 mai 2013, M. Jérôme P., cons. 8.

144. Ibid., cons. 9.

145. CC, déc. n 2013-331 QPC, cons. 10. Voir déjà en ce sens, CC, déc. nº 2012-280 QPC, cons. 16.

146. CC, déc. $\mathrm{n}^{\circ}$ 2013-331 QPC, cons. 12.

147. Déjà en ce sens, CC, déc. n 2013-327 QPC, cons. 5; CC, déc. 2012-287 QPC du 15 janvier 2013, Société française du radiotéléphone - SFR, cons. 3.

148. CC, déc. $\mathrm{n}^{\circ}$ 2013-327 QPC, cons. 6. Certains auteurs ont relevé que «le Conseil constitutionnel fait montre d'une certaine souplesse à l'égard du législateur en ce domaine. [...] L'ensemble des validations fiscales pourraient être considérées comme justifiées par un objectif d’intérêt général»; A. Perin-Dureau, Bulletin Joly mensuel d'information des sociétés, nº 12,2013, p. 841.

149. CC, déc. nº 2012-287 QPC, cons. 6 .

150. CC, déc. $\mathrm{n}^{\circ}$ 2013-675 DC, cons. 40.

151. CC, déc. n 2012-288 QPC du 17 janvier 2013, Consorts M., cons. 8.

152. CC, déc. no 2013-350 QPC du 25 octobre 2013, Commune du Pré-Saint-Gervais. 
presse et la liberté d'expression méconnaît les exigences de l'article 16 car les autorités publiques dotées de la personnalité morale autres que l'État ne peuvent obtenir réparation de leur préjudice résultant d'une diffamation que si l'action publique a été engagée par le ministère public devant le juge répressif. Il s'agit en effet ici, non d'une simple limitation, mais d'une violation substantielle du droit au recours ${ }^{153}$ le rendant quasiment inexistant en pratique. Comme le relève le commentaire aux Cahiers :

L'inconstitutionnalité dénoncée résulte en effet du cumul de la règle fermant l'action civile de la victime devant la juridiction civile et de celle imposant la mise en ouvre par le parquet de l'action publique devant la juridiction répressive ${ }^{154}$.

Le droit à un recours effectif n'implique toutefois pas celui de faire appel d'une décision de justice. Les articles L. 15-4 et L. 15-5 du Code de l'expropriation pour cause d'utilité publique sont dès lors validés par le Conseil bien qu'ils prévoient que l'indemnité fixée ne pourra être attaquée que par la voie du recours en cassation ${ }^{155}$. La solution est classique. Le Conseil avait déjà jugé que «le principe du double degré de juridiction n'a pas, en lui-même, valeur constitutionnelle ${ }^{156}$. En revanche, le droit au recours effectif induit, par principe, celui de se pourvoir en cassation, ce qu'a confirmé le Conseil dans sa décision $n^{\circ}$ 2013-314 QPC en abrogeant les mots «sans recours » de l'article 695-46 du Code de procédure pénale relatif au mandat d'arrêt européen ${ }^{157}$.

En outre, le Conseil a été amené à vérifier à plusieurs reprises en 2013 que les droits de la défense avaient bien été respectés, et cela dès le stade de l'instruction. Les droits de la défense supposent, tout d'abord, la possibilité d'être assisté le plus rapidement possible par un avocat. Toute atteinte à ce principe doit être proportionnée au but poursuivi, ce qui n'était pas le cas dans la décision $n^{\circ} 2013-679 D^{158}$. Le respect des droits de la défense impose, ensuite, celui du principe de loyauté dans l'administration de la preuve. Si le Conseil déclare conformes à la Constitution les articles 37 et 39 de la loi relative à la lutte contre la fraude fiscale et la grande délinquance économique et financière, il formule une réserve d'interprétation selon laquelle:

[...] les dispositions des articles 37 et 39 [...] ne sauraient, sans porter atteinte aux exigences découlant de l'article 16 de la Déclaration de 1789 , permettre aux services fiscaux et douaniers de se prévaloir de pièces ou documents obtenus par une autorité administrative ou judiciaire dans des conditions déclarées ultérieurement illégales par le juge ${ }^{159}$.

Enfin, découle traditionnellement de l'article 16 de la DDHC le respect de la garantie des droits. Le Conseil a rappelé, dans sa décision $\mathrm{n}^{\circ}$ 2013-685 DC que:

[...] si le législateur peut modifier rétroactivement une règle de droit, c'est à la condition de poursuivre un but d'intérêt général suffisant et de respecter tant les décisions de justice ayant force de chose jugée que le principe de non-rétroactivité des peines et des sanctions ${ }^{160}$.

Toutefois, et de manière classique ${ }^{161}$, il considère dans cette décision que la "petite rétroactivité fiscale» n'est pas contraire aux exigences de l'article $16^{162}$, étant inhérente à des impositions acquittées l'année suivant celle de la perception des revenus. La décision $\mathrm{n}^{\circ}$ 2013682 DC fait quant à elle une application extensive de la garantie des droits. Le Conseil affirme, dans une réserve d'interprétation, que la loi ne peut pas modifier les modalités d'imposition d'anciennes assurances-vies, censées être exonérées d'impôt sur le revenu du fait d'une certaine durée de détention. Pour le Conseil, «les contribuables ayant respecté cette durée de conservation pouvaient légitimement attendre l'application d'un régime particulier d'imposition lié au respect de cette durée légale ${ }^{163}$. C’est «le principe de "confiance légitime" qui est ainsi implicitement constitutionnalisé ${ }^{164}$.

153. Voir V. Saint-James, "La violation du droit au recours des collectivités territoriales en matière de diffamation", La semaine juridique, édition administrations et collectivités territoriales, $\mathrm{n}^{\circ}$ 50, 9 décembre 2013, chron. $\mathrm{n}^{\circ} 2357$.

154. Commentaire aux Cahiers du Conseil constitutionnel, p. 10 (disponible en ligne: http://www.conseil-constitutionnel.fr/conseil-constitutionnel/ root/bank/download/2013350QPCccc_350qpc.pdf).

155. CC, déc. $\mathrm{n}^{\circ}$ 2013-338/339 QPC, cons. 8 .

156. CC, déc. no 2004-491 DC du 12 février 2004, Loi complétant le statut de la Polynésie française, cons. 4; CC, déc. n²012-243/244/245/246 QPC du 14 mai 2012, Société Yonne Républicaine et autre, cons. 13.

157. CC, déc. no 2013-314 QPC du 14 juin 2013, M. Jeremy F., cons. 9: «qu'en privant les parties de la possibilité de former un pourvoi en cassation contre l'arrêt de la chambre de l'instruction statuant sur une telle demande, les dispositions contestées apportent une restriction injustifiée au droit à exercer un recours juridictionnel effectif; que, par suite, au quatrième alinéa de l'article 695-46 du Code de procédure pénale, les mots "sans recours" doivent être déclarés contraires à la Constitution".

158. Comme le relève le Conseil, «les infractions [...] de corruption et de trafic d'influence ainsi que de fraude fiscale et douanière, constituent des délits qui ne sont pas susceptibles de porter atteinte en eux-mêmes à la sécurité, à la dignité ou à la vie des personnes» (CC, déc. n $2013-679$ DC, cons. 77). Il est dès lors disproportionné d'appliquer ici la garde à vue exceptionnelle prévue par l'article 706-88 du Code pénal.

159. CC, déc. nº 2013-679 DC, cons. 33.

160. CC, déc. n 2013-685 DC, cons. 25.

161. Voir déjà par exemple CC, déc. $\mathrm{n}^{\circ}$ 2012-662 DC, cons. 108.

162. CC, déc. nº 2013-685 DC, cons. 26.

163. CC, déc. $\mathrm{n}^{\circ}$ 2013-682 DC du 19 décembre 2013, Loi de financement de la sécurité sociale pour 2014, cons. 17.

164. B. Mathieu, «Sécurité juridique - Le respect de la légitime confiance des citoyens s'impose au législateur », La semaine juridique, éd. G, nº 4 , 27 janvier 2014, doctr. n 116 